\title{
Measuring solar reflectance-Part II: review of practical methods
}

\author{
Ronnen Levinson \\ Hashem Akbari* \\ Paul Berdahl \\ Heat Island Group \\ Lawrence Berkeley National Laboratory
}

April 28, 2010

\begin{abstract}
A companion article explored how solar reflectance varies with surface orientation and solar position, and found that clear-sky air mass 1 global horizontal (AM1GH) solar reflectance is a preferred quantity for estimating solar heat gain. In this study we show that AM1GH solar reflectance $R_{\mathrm{g}, 0}$ can be accurately measured with a pyranometer, a solar spectrophotometer, or an updated version of the Solar Spectrum Reflectometer (version 6). Of primary concern are errors that result from variations in the spectral and angular distributions of incident sunlight.

Neglecting shadow, background and instrument errors, the conventional pyranometer technique can measure $R_{\mathrm{g}, 0}$ to within 0.01 for surface slopes up to $5: 12\left[23^{\circ}\right]$, and to within 0.02 for surface slopes up to $12: 12\left[45^{\circ}\right]$. An alternative pyranometer method minimizes shadow errors and can be used to measure $R_{\mathrm{g}, 0}$ of a surface as small as $1 \mathrm{~m}$ in diameter. The accuracy with which it can measure $R_{\mathrm{g}, 0}$ is otherwise comparable to that of the conventional pyranometer technique.

A solar spectrophotometer can be used to determine $R_{\mathrm{g}, 0}^{*}$, a solar reflectance computed by averaging solar spectral reflectance weighted with AM1GH solar spectral irradiance. Neglecting instrument errors, $R_{\mathrm{g}, 0}^{*}$ matches $R_{\mathrm{g}, 0}$ to within 0.006 . The air mass 1.5 solar reflectance measured with version 5 of the Solar Spectrum Reflectometer can differ from $R_{\mathrm{g}, 0}^{*}$ by as much as 0.08 , but the AM1GH output of version 6 of this instrument matches $R_{\mathrm{g}, 0}^{*}$ to within about 0.01 .
\end{abstract}

\footnotetext{
*Akbari's current address: Department of Building, Civil and Environmental Engineering, Concordia University, Montreal, Quebec, Canada.
} 


\section{Introduction}

In Part I of this study [1] we defined clear-sky air mass 1 global horizontal (AM1GH) solar reflectance $R_{\mathrm{g}, 0}$, a metric that can be used to accurately estimate the solar heat gain of an opaque surface. Here in Part II we consider the measurement of $R_{\mathrm{g}, 0}$ with each of three instruments: a pyranometer, a solar spectrophotometer and a Solar Spectrum Reflectometer. Of primary concern are errors that result from variations in the spectral and angular distributions of incident sunlight.

\section{Conventional pyranometer technique (method E1918)}

Global solar reflectance $R_{\mathrm{g}}$ can be measured with a pyranometer (solar radiation meter) by facing its sensor directly away from the target surface to measure incident global solar irradiance $I_{\mathrm{i}}$, then directly toward the target surface to measure reflected global solar irradiance $I_{\mathrm{r}}$. If preferred, $I_{\mathrm{i}}$ and $I_{\mathrm{r}}$ can also be measured simultaneously with back-to-back pyranometers. ASTM E1918-06 (Standard Test Method for Measuring Solar Reflectance of Horizontal and Low-Sloped Surfaces in the Field) [2] (hereafter, E1918) details the application of this technique to a surface whose pitch does not exceed 2:12 [9.5 $]$. The E1918 solar reflectance $R_{\mathrm{E} 1918} \equiv I_{\mathrm{r}} / I_{\mathrm{i}}$ will equal $R_{\mathrm{g}}$ if (a) the surface reflects diffusely; (b) the pyranometer casts no shadow on the surface; and (c) the pyranometer sees only the target surface when measuring $I_{\mathrm{r}}$. Simulations performed in Part I of this study indicate that for a horizontal surface or sun-facing low-sloped surface, $\left|R_{\mathrm{g}}-R_{\mathrm{g}, 0}\right| \leq 0.01$ when the solar zenith angle $z \leq 45^{\circ}$ (Figure 1). Therefore, under these ideal conditions $\left|R_{\mathrm{E} 1918}-R_{\mathrm{g}, 0}\right| \leq$ 0.01 .

This simple technique requires only a portable, relatively inexpensive instrument and applies equally well to flat and curved surfaces. However, there are some restrictions. First, the sky must be clear, particularly around the sun. Haze or cloudiness can change the spectral power distribution of sunlight, and the passage of a cloud across the sun can lead to serious error.

Second, the spectral distribution of $I_{\mathrm{i}}$ and the incidence angle $\theta$ of the solar beam both vary with hour of day and day of year. This can limit the daily time window during which $R_{\mathrm{E} 1918} \approx R_{\mathrm{g}, 0}$. For example, method E1918 requires that $z<45^{\circ}$. At a mid-U.S. latitude of $35^{\circ} \mathrm{N}$, this condition would be met from about 09:00 to 15:00 local standard time (LST) on June 21 (the summer solstice); about 10:00 to 14:00 LST on March 21 (the spring equinox) and September 21 (the autumn equinox); 
and not at all on December 21 (the winter solstice).

Third, the target must be large to ensure that nearly all reflected radiation collected by the downward-facing sensor comes from the target, rather than its surroundings. If a $3 \mathrm{~cm}$ diameter sensor is placed $50 \mathrm{~cm}$ above the center of a circular target, the target's diameter $\phi$ must be $3 \mathrm{~m}$ to yield a sensor-to-surface view factor $F$ of $0.90 ; 4.4 \mathrm{~m}$, for $F=0.95$; or $10 \mathrm{~m}$, for $F=0.99$ [3]. Thus when a $\phi=3 \mathrm{~m}$ white target of $R_{\mathrm{g}}=0.80$ is surrounded by a gray background of global solar reflectance 0.20 , the presence of the gray background will yield $R_{\mathrm{E} 1918}=0.74$, underestimating $R_{\mathrm{g}}$ by 0.06 . If the target were instead black with $R_{\mathrm{g}}=0.05$, the presence of the gray background would yield $R_{\mathrm{E} 1918}=0.065$, overestimating $R_{\mathrm{g}}$ by 0.015 . The need for a large target makes the method well suited to characterize roofs, pavements, lawns and other extensive surfaces, but inconvenient to apply to small samples like product prototypes or exposure coupons.

Fourth, this technique will always slightly underestimate $R_{\mathrm{g}}$ because the shadows cast by the pyranometer and its support reduce $I_{\mathrm{r}}$. However, this error can be fairly small if the support is designed to cast a minimal shadow. For example, consider a pyranometer $50 \mathrm{~cm}$ above the surface that casts a $15 \mathrm{~cm}$ diameter shadow concentric with its sensor when the sun is at zenith. The sensor-to-shadow view factor will be 0.022 . If $89 \%$ of the incident sunlight is beam radiation, shadow error will yield $R_{\mathrm{E} 1918}=0.98 \times R_{\mathrm{g}}$. Thus in the absence of background errors, $R_{\mathrm{g}}=0.80$ (white) will yield $R_{\mathrm{E} 1918}=0.784$, while $R_{\mathrm{g}}=0.05$ (black) will yield $R_{\mathrm{E} 1918}=0.049$.

Fifth, we note that pyranometer responsivity (signal/irradiance) is a strong function of the angle of incidence. Modern calibration methods model responsivity as function of this angle rather than as a fixed value [4].

A pyranometer can also be used to estimate $R_{\mathrm{g}}$ of an in-place pitched surface. The beam will be normal to the surface $(\theta=0)$ when the surface tilt angle $\Sigma=z$ and the surface solar azimuth

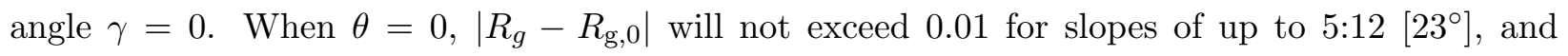
not exceed 0.02 for slopes up to 12:12 [45] (Figure 2). The surface can be rotated azimuthally away from the solar beam by up to $60^{\circ}$ without increasing the error beyond these limits (Figure 3 ). Thus, neglecting background, shadow and instrument errors, $\left|R_{\mathrm{E} 1918}-R_{\mathrm{g}, 0}\right|$ will not exceed 0.01 for slopes of up to 5:12, and not exceed 0.02 for slopes up to $12: 12$, so long as $\Sigma=z$ and $|\gamma| \leq 60^{\circ}$. 


\section{Alternative pyranometer technique (method E1918A)}

We have developed an alternative pyranometer-based technique that can estimate $R_{\mathrm{g}}$ of a diffusely reflective surface as small as $1 \mathrm{~m}$ in diameter. In this non-ASTM method "E1918A" [5], the pyranometer sensor is faced directly toward the target surface for three sequential measurements of $I_{\mathrm{r}}$. In the first configuration $(j=1)$, the target is exactly covered with a solar-opaque white

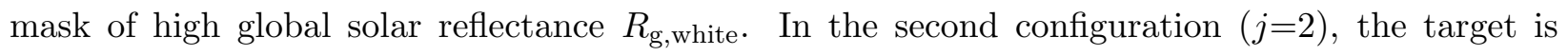

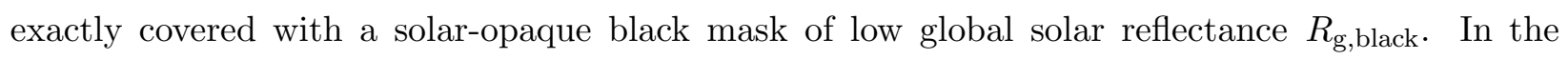
third configuration $(j=3)$, the surface is uncovered. Each $I_{\mathrm{r}, j}$ is proportional to the sum of the view-factor-weighted global solar reflectances of the target or mask and its surroundings. If $I_{\mathrm{i}}$ is constant, target $R_{\mathrm{g}}$ can be interpolated from $R_{\mathrm{g}, \text { black }}$ and $R_{\mathrm{g}, \text { white }}$. That is,

$$
R_{\mathrm{E} 1918 \mathrm{~A}} \equiv R_{\mathrm{g}, \mathrm{black}}+\frac{I_{\mathrm{r}, 3}-I_{\mathrm{r}, 2}}{I_{\mathrm{r}, 1}-I_{\mathrm{r}, 2}}\left(R_{\mathrm{g}, \text { white }}-R_{\mathrm{g}, \mathrm{black}}\right)
$$

Each mask should ideally be a nonselective and diffuse reflector to minimize the variation of its reflectance with solar position.

Like E1918, method E1918A uses a portable, relatively inexpensive instrument and applies equally well to flat and curved surfaces. Each technique requires a clear sky and a solar position that yields $\theta<45^{\circ}$. However, method 1918A can be applied to much smaller targets because the only component of $I_{\mathrm{r}}$ that changes over the course of the three measurements is that reflected from the target or mask. In theory, the target can be vanishingly small. In practice, reducing the target diameter $\phi$ decreases the sensor-to-target view factor $F$, making $R_{\mathrm{E} 1918 \mathrm{~A}}$ more sensitive to errors in measurement of $I_{\mathrm{r}}$. For example, consider the use of masks with $R_{\mathrm{g}, \text { black }}=0.05$ and $R_{\mathrm{g}, \text { white }}=0.80$. If $I_{\mathrm{i}}=1000 \mathrm{~W} \mathrm{~m}^{-2}$, the white-black difference in reflected irradiance $\Delta I_{\mathrm{r}} \equiv I_{\mathrm{r}, 1}-I_{\mathrm{r}, 2}$ will be $600 \mathrm{~W} \mathrm{~m}^{-2}$ for $\phi=2 \mathrm{~m}(F=0.8) ; 375 \mathrm{~W} \mathrm{~m}^{-2}$ for $\phi=1 \mathrm{~m}(F=0.5)$; or $150 \mathrm{~W} \mathrm{~m}^{-2}$ for $\phi=0.5 \mathrm{~m}$ $(F=0.2)$. A $10 \mathrm{~W} \mathrm{~m}^{-2}$ uncertainty in measurement of $I_{\mathrm{r}}$ would represent $1.7 \%, 2.7 \%$ or $6.7 \%$ of these three values of $\Delta I_{\mathrm{r}}$. We compromise between accuracy and convenience by recommending a minimum target diameter of about $1 \mathrm{~m}$.

The presence of the pyranometer's shadow on the target will cause little to no error in measurement of $R_{\mathrm{E} 1918 \mathrm{~A}}$. If $R_{\mathrm{g}}, R_{\mathrm{g}, \text { black }}$ and $R_{\mathrm{g}, \text { white }}$ do not change when the target is moved from full sun 
to shade, there will be no shadow error. Otherwise, the shadow error will be very small because the contribution of the shaded area to $I_{\mathrm{r}}$ is minimal. For example, when $z=0$, global horizontal solar irradiance in shade is about $11 \%$ of that in full sun, and the view factor from the sensor to the pyranometer shadow is 0.022 (c.f. $\S 2$ ). Hence, when full-sun $I_{\mathrm{i}}$ is $1000 \mathrm{~W} \mathrm{~m}^{-2}$, a 0.30 difference between full-sun and shade values of $R_{\mathrm{g}}$ would yield a $0.7 \mathrm{~W} \mathrm{~m}^{-2}$ shadow error in measurement of $I_{\mathrm{r}}$. This in turn would represent $0.2 \%$ of $\Delta I_{\mathrm{r}}=375 \mathrm{~W} \mathrm{~m}^{-2}$ for $\phi=1 \mathrm{~m}$.

In method 1918A, one can also position the pyranometer to move its shadow off the target. This will eliminate shadow error but reduce $F$. The distance by which the vertical axis of the pyranometer must be offset from the vertical axis of the target, and the consequent reduction in $F$, depends on $z$. Consider a 15 -cm diameter pyranometer with a 3 -cm diameter sensor placed $50 \mathrm{~cm}$ above a 1-m diameter concentric target. $F$ in this zero-offset configuration is 0.50 . When $z=30^{\circ}$, the pyranometer must be horizontally offset $29 \mathrm{~cm}$ to move its shadow off the target, reducing $F$ to 0.42 . When $z=0$, the offset must about $58 \mathrm{~cm}$, reducing $F$ to 0.22 . With these view factors of $0.50,0.42$ and 0.22 , a $10 \mathrm{~W} \mathrm{~m}^{-2}$ uncertainty in measurement of $I_{\mathrm{r}}$ would represent $2.7 \%, 3.2 \%$, or $6.1 \%$ of $\Delta I_{\mathrm{r}}$.

\section{Solar spectrophotometer (method E903)}

A solar spectrophotometer illuminates a surface with monochromatic light at near-normal incidence and measures light reflected into an integrating sphere. A series of such measurements at wavelengths spanning the solar spectrum $(300$ - $2500 \mathrm{~nm})$ yields the surface's near-normal beamhemispherical solar spectral reflectance $r_{\mathrm{b}, \mathrm{nn}}(\lambda)$. While wavelength spacing is arbitrary, we find measuring solar spectral reflectance at a $5 \mathrm{~nm}$ interval sufficient to capture the spectral details of most surfaces.

A properly calibrated solar spectrophotometer can accurately measure $r_{\mathrm{b}, \mathrm{nn}}(\lambda)$, but there are several limits to its use. First, the beam typically illuminates only about $10 \mathrm{~mm}^{2}$ of the sample. Since each solar spectral reflectance measurement series can take several minutes, this instrument is most convenient to apply to a spatially uniform sample that can be characterized by a single measurement series. Second, the beam angle is typically fixed near normal $\left(\theta \approx 8^{\circ}\right)$, making it difficult to measure reflectance at far-from-normal incidence. Third, the sample must be flat, 
to ensure that any specularly reflected light is captured by the integrating sphere; small enough (typically not larger than $10 \mathrm{~cm} \times 15 \mathrm{~cm}$ ) to fit in the instrument's sample port; and, if the instrument has a vertical sample port, sufficiently cohesive to be mounted vertically. Fourth, a solar spectrophotometer is a large, immobile and expensive bench instrument to which samples must be brought. This may require destructive extraction of coupons from large surfaces.

Solar reflectance can be computed as the mean of solar spectral reflectance weighted by solar spectral irradiance. We showed in Part I of this study that (a) $r_{\mathrm{b}, \mathrm{nn}}(\lambda)$ at $\theta=8^{\circ}$ equals normalincidence beam-hemispherical solar spectral reflectance $r_{\mathrm{b}, \mathrm{n}}(\lambda)$ to within $10^{-5} ;(\mathrm{b})$ averaging $r_{\mathrm{b}, \mathrm{n}}(\lambda)$ weighted by normal-incidence beam solar spectral irradiance ("direct-normal" solar spectral irradiance) $i_{\mathrm{b}, \mathrm{n}}(\lambda)$ yields normal-incidence beam-hemispherical solar reflectance $R_{\mathrm{b}, \mathrm{n}}$, also known as direct-normal solar reflectance; and (c) averaging $r_{\mathrm{b}, \mathrm{n}}(\lambda)$ weighted by AM1GH solar spectral irradiance $i_{\mathrm{g}, 0}(\lambda)$ yields a metric $R_{\mathrm{g}, 0}^{*}$ that approximates $R_{\mathrm{g}, 0}$ to within 0.006 .

ASTM E903-96 (Standard Test Method for Solar Absorptance, Reflectance, and Transmittance of Materials Using Integrating Spheres) [6] (hereafter, E903) details the measurement of $r_{\mathrm{b}, \mathrm{nn}}(\lambda)$ using a spectrophotometer equipped with an integrating sphere. This method is currently withdrawn but remains useful for measuring solar spectral reflectance.

Method E903 specifies that the solar reflectance of a terrestrial surface should be calculated by averaging $r_{\mathrm{b}, \mathrm{nn}}(\lambda)$ weighted with the air mass 1.5 beam normal solar spectral irradiance described in ASTM E891-87 (Tables for Terrestrial Direct Normal Solar Spectral Irradiance for Air Mass 1.5) [7]. The E891BN (E891 Beam Normal) irradiance $i_{\mathrm{E} 891 \mathrm{BN}}(\lambda)$ was designed to represent light incident on the receiver of a solar concentrator, a solar collector illuminated by multiple sun-tracking mirrors. Hence, it includes only beam sunlight. An air mass of 1.5 was selected by determining that in many U.S. locations, about half of the annual beam-normal solar energy is delivered when the absolute (pressure corrected) air mass is less than or equal to 1.5, and about half when the air mass is above 1.5. The atmosphere was assumed to have an aerosol optical depth (AOD) of 0.270 at $500 \mathrm{~nm}$, intended as an average value for the continental United States [8].

ASTM has since withdrawn Standard E891 in favor of the air mass 1.5 beam normal irradiance detailed in ASTM G173-03 (Standard Tables for Reference Solar Spectral Irradiances: Direct Normal and Hemispherical on $37^{\circ}$ Tilted Surface) [9]. The primary difference between ASTM E891 and ASTM G173 in modeling beam-normal solar spectral irradiance is that the latter assumes an 
AOD of 0.084 , rather than 0.270 . The reduced AOD is intended to represent an average value for the southwestern U.S., the "sunbelt" in which solar concentrators are likely to be deployed $[8,9,10,11]$. For convenience, we will refer to the ASTM G173 atmosphere (AOD 0.084) as a clear sky, and the ASTM E891 atmosphere (AOD 0.270) as a hazy sky. The beam normal and global tilt solar irradiances specified by ASTM G173 are denoted G173BN and G173GT, respectively.

Method E903 is appropriate for measuring $r_{\mathrm{b}, \mathrm{nn}}(\lambda)$. However, there are several difficulties with its procedure for calculating the solar reflectance of a terrestrial surface. First, it describes the calculation of $R_{\mathrm{b}, \mathrm{n}}$, rather than $R_{\mathrm{g}} . \quad R_{\mathrm{b}, \mathrm{n}}$ is an excellent metric for a solar concentrator, which receives about $99 \%$ beam sunlight at 10 suns, and about $99.9 \%$ beam sunlight at 100 suns. $^{1}$ However, it is not a suitable metric for a surface illuminated by unconcentrated sunlight, which is only about $89 \%$ beam radiation under AM1GH conditions (a horizontal surface under a clear sky illuminated by one sun at solar zenith).

Second, air mass 1.5 was chosen specifically for a solar concentrator, such as a collector illuminated almost exclusively by beam sunlight from sun-tracking mirrors [8]. This particular air mass value is not generally representative of the sunlight received by a fixed surface, even one illuminated only by beam sunlight. For example, the ratio of the beam sunlight incident on a horizontal surface to that incident on a sun-tracking (beam-normal) surface equals cos $z$. Since the beam solar irradiance on a horizontal surface will decline more rapidly with increasing air mass than that incident on a sun-tracking surface, the air mass splitting the annual beam normal solar energy received by a horizontal surface will be less than 1.5.

Third, since E903 solar spectral reflectance is measured at near-normal incidence, it should be weighted by near-normal solar spectral irradiance to calculate solar reflectance at near-normal incidence. Calculating the global solar reflectance of a horizontal surface from $r_{\mathrm{b}, \mathrm{nn}}(\lambda)$ requires weighting the reflectance with $i_{\mathrm{g}}(\lambda)$ evaluated near zenith (near air mass 1 ). At air mass 1.5, the solar beam is near-normal only to a sun-facing surface tilted about $48^{\circ}$ from horizontal. Therefore, we recommend reserving the use of an air mass 1.5 beam-normal solar spectral irradiance for its intended purpose - estimating the annual energy output of a solar concentrator.

\footnotetext{
${ }^{1}$ Say unconcentrated global sunlight of irradiance $I_{1}=1 \mathrm{~kW} \mathrm{~m}^{-2}$ is about $90 \%$ beam $\left(0.9 \mathrm{~kW} \mathrm{~m}^{-2}\right)$ and $10 \%$ diffuse $\left(0.1 \mathrm{~kW} \mathrm{~m}^{-2}\right)$. Then concentration to 10 suns $\left(I_{10}=10 \times I_{1}=10 \mathrm{~kW} \mathrm{~m}^{-2}\right)$ through the addition of focused beam sunlight will yield an irradiance that is about $99 \%$ beam $\left(9.9 \mathrm{~kW} \mathrm{~m}^{-2}\right)$ and $1 \%$ diffuse $\left(0.1 \mathrm{~kW} \mathrm{~m}^{-2}\right)$. Similarly, concentration to 100 suns $\left(I_{100}=100 \times I_{1}=100 \mathrm{~kW} \mathrm{~m}^{-2}\right)$ will yield an irradiance that is about $99.9 \%$ beam $(99.9$ $\left.\mathrm{kW} \mathrm{m} \mathrm{m}^{-2}\right)$ and $0.1 \%$ diffuse $\left(0.1 \mathrm{~kW} \mathrm{~m}^{-2}\right)$.
} 
Fourth, E903 specifies that terrestrial solar reflectance is to be calculated using a "50-point selected ordinate method" in which the solar irradiance distribution is divided into $n$ wavelength intervals each containing $1 / n$ of the total irradiance. Solar reflectance is then computed as the mean of the spectral reflectances at the interval centroids. If one measures solar spectral reflectance at fixed intervals (e.g., 300 to $2500 \mathrm{~nm}$ at a $5 \mathrm{~nm}$ interval) to capture spectral features, it is more straightforward to calculate solar reflectance by weighting the fixed-interval solar spectral reflectance with solar spectral irradiance evaluated at the same wavelengths. E903 specifies this "weighted ordinate method" only for computing extraterrestrial solar reflectance. We suggest that both selected and weighted ordinate methods should be allowed.

Fifth, the NIR fractions of the E891BN hazy-sky beam normal solar irradiance (58.1\%) and the G173BN clear-sky beam normal solar irradiance (54.3\%) are both higher than that of AM1GH solar irradiance (48.7\%); see Figure 4. Using either of these beam-normal solar spectral irradiances as a weighting function can lead to large errors in the global solar reflectance of a spectrally selective surface. For example, in Part I of this study we demonstrated that (a) the AM1GH solar reflectance of a selective ("cool") black surface computed by averaging $r_{\mathrm{b}, \mathrm{nn}}(\lambda)$ weighted by $i_{\mathrm{g}, 0}(\lambda)$ is 0.46 ; and that (b) weighting $r_{\mathrm{b}, \mathrm{nn}}(\lambda)$ with $i_{\mathrm{G} 173 \mathrm{BN}}(\lambda)$ or $i_{\mathrm{E} 891 \mathrm{BN}}(\lambda)$ yields a solar reflectance of $0.51(0.05$ higher) or 0.54 (0.08 higher), respectively.

There are two other active standards for the measurement of solar spectral reflectance, each of which yields a beam-normal solar reflectance. ANSI/ASHRAE Standard 74-1988 [12] weights solar spectral reflectance with an early version of E891BN (from ASTM E891-82 [13]) to calculate terrestrial solar reflectance. ASTM Standard E424-71(2007) [14] describes in substantially less detail than E903 the measurement of solar spectral reflectance and transmittance using a spectrophotometer with an integrating sphere. E424 uses the year-1940 Parry Moon AM2 beam-normal solar spectral irradiance [15] as a weighting function to calculate solar properties from spectral properties. Its procedure for calculating solar reflectance is unsuitable for roofs and pavements for much the same reasons that apply to the use of E891BN in E903.

We address these concerns by defining non-ASTM method "E903G" (E903 Global) for using a solar spectrophotometer equipped with an integrating sphere to determine $r_{\mathrm{b}, \mathrm{nn}}(\lambda)$ and $R_{\mathrm{g}, 0}^{*}$. In E903G, one follows method E903 to measure $r_{\mathrm{b}, \mathrm{nn}}(\lambda)$ over the solar spectrum (300 to $2500 \mathrm{~nm}$ ) at a $5 \mathrm{~nm}$ interval. $R_{\mathrm{g}, 0}^{*}$ is then calculated by averaging $r_{\mathrm{b}, \mathrm{nn}}(\lambda)$ weighted by $i_{\mathrm{g}, 0}(\lambda)$. We tabulate 
$i_{\mathrm{g}, 0}(\lambda)$ in an online archive [16].

It was shown in Part I that $R_{\mathrm{g}, 0}^{*}$ will exactly match $R_{\mathrm{g}, 0}$ if a surface is matte, and slightly underestimate $R_{\mathrm{g}, 0}$ if the surface is glossy. This underestimation will not exceed 0.006 for a surface of typical real refractive index 1.5. Hence, we seek to include the E903G procedure for computing AM1GH solar reflectance in the next revision to E903.

In future work, goniophotometric measurements of bi-directional solar spectral reflectance [17] could be used to examine the relationship between near-normal and hemispherical reflectances.

\section{Solar Spectrum Reflectometer (method C1549)}

The diffuse-directional spectral reflectance of a surface is equal to its directional-hemispherical spectral reflectance [3]. A hemispherical reflectometer uses this principle to indirectly measure near normal-hemispherical reflectance by illuminating a surface with diffuse light and sensing light reflected at near-normal incidence. The Solar Spectrum Reflectometer (SSR) made by Devices \& Services (Dallas, TX) [18] is a hemispherical reflectometer designed to measure near normalhemispherical solar reflectance.

In the SSR, the diffuse light source is a white chamber illuminated by a tungsten lamp. The surface to be characterized is placed at a $2.5 \mathrm{~cm}$ diameter aperture in the chamber wall where it is shielded from the lamp by a baffle. Four separate detectors view light reflected from the surface through a shared collimating tube angled $20^{\circ}$ from the surface's normal. Each detector is a silicon or lead sulfide light sensor covered with a set of spectrally selective light filters. The spectral response of each detector to the spectral reflectance of the surface is equal to the product of the spectral radiosity of the lamp, the spectral reflectance of the chamber wall, the spectral transmittance of its filter set and the spectral sensitivity of its sensor. Standards of known reflectance are used to correct for drifts in response that can result from aging or soiling of the apparatus. The four detectors are named L1 (IR), L2 (Red), L3 (Blue), and L4 (UV), where each parenthetical description roughly locates the peak of the detector's spectral response. (The IR peak is in the near infrared, rather than in the thermal infrared.)

Since diffuse-near normal spectral reflectance equals $r_{\mathrm{b}, \mathrm{nn}}(\lambda)$, the signal $Y_{j}$ returned by detector $j$ is the integral over all wavelengths $\lambda$ of the product of $r_{\mathrm{b}, \mathrm{nn}}(\lambda)$ and the detector's spectral response 
$f_{j}(\lambda)$. Let $F_{j}$ represent the integral over all wavelengths of $f_{j}(\lambda)$. The SSR reports for each detector a mean surface reflectance $D_{j}=Y_{j} / F_{j}$ that equals the average of $r_{\mathrm{b}, \mathrm{nn}}(\lambda)$ weighted with $f_{j}(\lambda)$.

It can be shown that when the four detector reflectances $D_{j}$ are totaled with weights $c_{j}=$ $a_{j} F_{j} / \sum_{k} a_{k} F_{k}$, the sum $R=\sum_{j} c_{j} D_{j}$ will equal the average of $r_{\mathrm{b}, \mathrm{nn}}(\lambda)$ weighted with an aggregate spectral response $g(\lambda)=\sum_{j} a_{j} f_{j}(\lambda)$. If the spectral response functions $f_{j}(\lambda)$ and their multipliers $a_{j}$ are designed to yield an aggregate spectral response $g(\lambda)$ that is exactly proportional to solar spectral irradiance $i(\lambda), R$ will equal the surface's near normal beam-hemispherical solar reflectance, $S$.

The SSR model in wide release in 2008 (version 5, abbreviated SSRv5) reports eight reflectances. The first four values are the detector reflectances $D_{1} \ldots D_{4}$, displayed as L1...L4. The second four values, named AM0, AM1, AM1.5, and AM2, are near-normal beam-hemispherical solar reflectances evaluated with solar (aggregate) spectral responses that approximate beam normal solar spectral irradiances at air masses 0, 1, 1.5 and 2. The AM0 and AM1 solar spectral responses are modeled after air-mass 0 and air-mass 1 beam normal solar spectral irradiances given by Thekaekara [19]. The AM1.5 solar spectral response simulates $i_{\mathrm{E} 891 \mathrm{BN}}(\lambda)$, and basis of the AM2 solar spectral response is no longer known [20].

Each solar spectral response $g(\lambda)$ was designed to make its power fractions in five spectral bands (B1, 300 - $400 \mathrm{~nm} ; \mathrm{B} 2,400$ - $600 \mathrm{~nm}$; B3, 600 - $900 \mathrm{~nm}$; B4, 900 - $1400 \mathrm{~nm}$; and B5, 1400 - 2500 $\mathrm{nm})$ closely match those in the solar spectral irradiance $i(\lambda)$ being approximated [21]. However, an investigation by the manufacturer and Berkeley Lab has revealed that the L2 (Red) detector in SSRv5 is more responsive to NIR light than intended. This makes the SSRv5's actual solar spectral responses more NIR sensitive than intended. To illustrate, Figure 5 compares the nominal and actual SSRv5 AM1.5 solar spectral responses to $i_{\mathrm{E} 891 \mathrm{BN}}(\lambda)[22]$. Each band power fraction in the nominal $g(\lambda) \operatorname{SSRv5\_ AM1.5\_ nom~matches~that~in~} i_{\mathrm{E} 891 \mathrm{BN}}(\lambda)$ to within $0.8 \%$ (absolute). However, band power fractions in the actual $g(\lambda)$ differ from those in the $i_{\mathrm{E} 891 \mathrm{BN}}(\lambda)$ by as much as $4.3 \%$ (absolute). In particular, the B3 (600 - $900 \mathrm{~nm})$ and B4 (900 - $1400 \mathrm{~nm})$ fractions are $0.9 \%$ and $4.3 \%$ high (absolute), while the B2 (400 - $600 \mathrm{~nm}$ ) fraction is $2.3 \%$ low (absolute). This tends to make the SSRv5_AM1.5 solar reflectance of a cool color-a surface whose NIR reflectance exceeds its visible reflectance-higher than intended. 
We describe the amount by which a surface's E903_AM1GH near-infrared reflectance $N$ exceeds its E903G_AM1GH visible reflectance $V$ as its "selectance," $\sigma=N-V$. Selectance can theoretically range from -1 to +1 , though we do not expect $\sigma$ to reach either extreme. Conventionally colored surfaces usually have $\sigma$ near zero (about -0.1 to +0.1 ), while cool colors tend to have $\sigma$ much greater than zero (about +0.1 to +0.6 for most cool colors, and up to about +0.85 for a high-performance cool black). One can also create a surface with $\sigma$ as low as about -0.2 by using a pigment, such as titanium dioxide rutile white, that when incorporated in a coating exhibits exceptionally strong visible backscattering.

We compiled a set of 125 samples with which to compare reflectometer solar reflectances to spectrophotometer solar reflectances. This reference set includes

- 103 glazed, mostly cool-colored ceramic tiles (shades of blue, green, yellow, orange, red, gray, and brown; $\sigma=+0.08$ to +0.56 ) and one unglazed ceramic tile (white; $\sigma=+0.07$ ) supplied by the Ferro Corporation;

- seven conventionally colored (black, blue, dark green, light green, red and gray; $\sigma=+0.01$ to $+0.13)$ and seven visually matched cool-colored $(\sigma=+0.23$ to +0.35$)$ factory-coated metal panels supplied by BASF Industrial Coatings; and

- one nonselective black (iron-oxide black, $\sigma=0.00$ ) and six "warm" gray (iron-oxide black tinted with titanium dioxide rutile white; $\sigma=-0.04$ to -0.14 ) hand-painted metal panels prepared by Berkeley Lab.

The solar spectral reflectance $r_{\mathrm{b}, \mathrm{nn}}(\lambda)$ of each sample was measured at Berkeley Lab with a PerkinElmer Lambda 900 UV/VIS/NIR spectrophotometer equipped with a 150-mm Labsphere integrating sphere. Spectral reflectances were measured from 300 to $2500 \mathrm{~nm}$ in $5 \mathrm{~nm}$ steps. The selectance distribution of the reference set is shown in Figure 6.

Figure 7 illustrates the increase with $\sigma$ of the amount by which the solar reflectance expected from the actual SSRv5_AM1.5 $g(\lambda)$ exceeds that expected from the nominal SSRv5_AM1.5 $g(\lambda)$. For a cool color with $\sigma=+0.4$, this difference is about +0.01 . However, the absolute accuracy with which solar reflectance expected from the actual SSRv5_AM1.5 $g(\lambda)$ matches E903_E891BN solar reflectance (Figure 8) is comparable to that which solar reflectance expected from the nominal 
SSRv5_AM1.5 $g(\lambda)$ matches E903_E891BN solar reflectance (Figure 9). That is, solar reflectances expected from the actual $g(\lambda)$ tend to slightly overpredict E903_E891BN solar reflectance, while those expected from the nominal $g(\lambda)$ tend to slightly underpredict E903_E891BN solar reflectance.

Figure 10 plots for an instrument operated by Devices \& Services the variation with $\sigma$ of the amount $\Delta S$ by which SSRv5_AM1.5 solar reflectance exceeds E903_E891BN solar reflectance. The performance of this unit (\#137) happens to be quite close to that expected from the actual SSRv5_AM1.5 $g(\lambda)$ (Figure 11). However, the SSRv5 solar reflectance of a selective sample can vary significantly from instrument to instrument.

Figure 12 shows the $\sigma$ distribution of the seven conventionally colored and seven cool colored factory-coated metal panels in the reference set. In a round-robin comparison led by Berkeley Lab, the reflectances of these 14 metal panels were measured with eight different SSRv5 instruments. Some instruments ran cool, yielding for surfaces with high $\sigma$ an SSRv5_AM1.5 solar reflectance higher than that expected from the actual SSRv5_AM1.5 spectral response, while others ran warm, yielding for surfaces with high $\sigma$ an SSRv5_AM1.5 solar reflectance lower than that expected (Figure 13).

Excluding some outliers for instrument \#087 that the manufacturer attributes to an intermittent firmware error, the difference $\Delta S$ between measured and expected values of SSRv5_AM1.5 solar reflectance for the round-robin sample set ranged from about -0.010 to +0.015 . We expect that the difference range would be greater if the round-robin set included samples with selectance greater than +0.35 . For example, the linear fits of $\Delta S$ to $\sigma$ shown in Figure 13 indicate that the difference could be as great as -0.015 to +0.025 for a cool colored surface with $\sigma=+0.6$. This suggests that any bias in SSRv5_AM1.5 solar reflectance induced by prior mischaracterization of the L2 sensor response could easily be masked in an instrument that runs warm.

Of greater concern when using the SSRv5 to measure the solar reflectance of anything other than the receiver of a solar concentrator is the mismatch between the SSRv5_AM1.5 $g(\lambda)$ and $i_{\mathrm{g}, 0}(\lambda)$ (Figure 14). This discrepancy stems largely from the difference between the shape of the 58\%-NIR E891BN $g(\lambda)$ and that of the 48\%-NIR AM1GH $i(\lambda)$ (Figure 4). Compared to $i_{\mathrm{g}, 0}(\lambda)$, the B3 (600 - $900 \mathrm{~nm})$ and B4 (900 - $1400 \mathrm{~nm})$ band power fractions of the SSRv5_AM1.5 $g(\lambda)$ are 3.7\% and 8.3\% high (absolute), while the B1 (300 - $400 \mathrm{~nm})$ and B2 (400 - $600 \mathrm{~nm})$ fractions are 3.9\% and $8.3 \%$ low (absolute). Figure 15 plots for the full reference set the variation with $\sigma$ of the amount 
$\Delta S$ by which SSRv5_AM1.5 solar reflectance measured with instrument \#137 exceeds $R_{\mathrm{g}, 0}^{*} . \Delta S$ ranges from about -0.02 to +0.085 , with mean values near zero for conventional colors, 0.05 for cool colors, and -0.02 for warm grays.

Devices \& Services has prepared a version 6 of the SSR that can measure several solar reflectances not available in version 5 [22]. The upgrade to version 6 from version 5 includes several new features. It (a) replaces an infrared filter to allow the spectral response of L1 to better match NIR solar spectral irradiance; (b) introduces two virtual detectors, L5 and L6, that are the signals from detectors L1 and L2 measured when the lamp's color temperature has been reduced to $2300 \mathrm{~K}$ from $3125 \mathrm{~K}$; (c) recharacterizes the spectral response of each detector to create more accurate solar spectral responses; and (d) outputs seven new solar reflectances-AM1GH, AM1BN, AM1DH, AM0BN, G173GT, G173BN and E891BN—while also emulating the AM0, AM1, AM1.5 and AM2 solar reflectances native to version 5. That is, while version 5 has four detectors (L1v5...L4v5) and reports four solar reflectances (SSRv5_AM0, SSRv5_AM1, SSRv5_AM1.5 and SSRv5_AM2), version 6 has six detectors (L1v6...L6v6) and reports 11 solar reflectances (new values SSRv6_AM1GH, SSRv6_AM1BN, SSRv6_AM1DH, SSRv6_AM0BN, SSRv6_G173GT, SSRv6_G173BN and SSRv6_E891BN, plus emulations SSRv6_V5AM0, SSRv6_V5AM1, SSRv6_V5AM1.5 and SSRv6_V5AM2).

It is also possible to estimate the seven new solar reflectances AM1GH, AM1BN, AM1DH, AM0BN, G173GT, G173BN and E891BN with SSRv5 if version 6 is not available. In this approach, one sums the version 5 detector reflectances L1v5...L4v5 with detector weights chosen to create SSRv5 auxiliary ("aux") $g(\lambda)$ that mimic each new $i(\lambda)$ (Table 1). Figure 16 compares to each new spectral irradiance $n$ the corresponding SSRv6_ $n$ and SSRv5_n_aux spectral responses. For each $i(\lambda)$ other than AM1DH, the band power fractions of the SSRv6 $g(\lambda)$ match those of the corresponding $i(\lambda)$ to within an absolute error not exceeding $2.3 \%$, and typically closer to $1 \%$. Again excluding AM1DH, the matches offered by the SSRv5 auxiliary $g(\lambda)$ are looser, with band power fraction absolute errors as large as 5.8\%. Version 6 can match these six irradiances more closely than can version 5 because it has both a new IR filter and a pair of virtual detectors with which to shape its aggregate responses. Neither SSRv6 nor SSRv5 auxiliary offers a particularly tight match to AM1DH because the shape of the AM1DH $i(\lambda)$ differs from those of the other six irradiances (Figure 17). 
The $g(\lambda)$ of each emulation SSRv6_V5AM0, SSRv6_V5AM1, SSRv6_V5AM1.5 and SSRv6_V5AM2 closely matches that of its corresponding SSRv5 $g(\lambda)$, with band power absolute errors not exceeding $1.2 \%$ (Figure 16). The greatest differences are in the B4 band (900 - $1400 \mathrm{~nm})$, where the replacement of the IR filter has changed the shape of the L1 spectral response.

Figures 18 and 19 plot for each new irradiance $n$ the variation with $\sigma$ of the amount $\Delta S$ by which the SSRv6_n or SSRv5_n_aux solar reflectance measured with instrument \#137 exceeds the corresponding E903_n solar reflectance. Using SSRv6, the range of $\Delta S$ for the full reference set is -0.012 to 0.020 for AM1DH, and within -0.013 to +0.013 for the other six irradiances. As expected from the looser matches of each SSRv5_aux $g(\lambda)$ to each new $i(\lambda), \Delta S$ is generally greater for version 5 , ranging from -0.035 to +0.017 . The difference $\Delta S$ is uncorrelated with selectance for both SSRv6 and SSRv5_aux (coefficient of determination $R^{2} \leq 0.03$ ).

Figure 20 plots for each SSRv5 native solar reflectance $n=\mathrm{AM}$, AM1, AM1.5 or AM2 the amount by which the emulation solar reflectance SSRv6_V5n measured with instrument \#137 exceeds the SSRv5_n solar reflectance measured with the same instrument. The range of $\Delta S$ for the full reference set is within -0.005 to +0.006 for each solar reflectance, and the difference $\Delta S$ is uncorrelated with $\sigma\left(R^{2}<0.01\right)$. This suggests that the SSRv6 can accurately emulate SSRv5 when necessary.

We recommend using version 6 of the SSR to accurately estimate $R_{\mathrm{g}, 0}^{*}$ to within about \pm 0.01 . However, if only version 5 is available, the auxiliary solar reflectance SSRv5_AM1GH_aux can be used to estimate $R_{\mathrm{g}, 0}^{*}$ to within about -0.03 to +0.01 . As noted in the previous section, $R_{\mathrm{g}, 0}^{*}$ will underestimate $R_{\mathrm{g}, 0}$ by no more than 0.006 .

\section{Conclusions}

AM1GH solar reflectance $R_{\mathrm{g}, 0}$ can be measured with a conventional pyranometer method (ASTM E1918), an alternative pyranometer method (non-ASTM method E1918A), a solar spectrophotometer (non-ASTM method E903G) or a Solar Spectrum Reflectometer (variant on ASTM C1549).

Under ideal conditions, the conventional pyranometer method can determine $R_{\mathrm{g}, 0}$ of a low sloped surface (pitch $\leq 2: 12\left[9.5^{\circ}\right]$ ) to within 0.01 . It can also measure $R_{\mathrm{g}, 0}$ of a pitched surface to within 0.01 for slopes up to $5: 12\left[23^{\circ}\right]$, and to within 0.02 for slopes up to $12: 12$ [45] , so long as 
the solar beam is approximately normal to the surface. These small errors result from the spectral and angular variation of solar reflectance. Shadow errors can lead this method to underestimate solar reflectance by a few percent of its true value. To minimize background error, this method is best applied to extensive surfaces like roofs, lawns and pavements.

The alternative pyranometer method minimizes shadow errors and can be used to measure $R_{\mathrm{g}, 0}$ of a target as small as $1 \mathrm{~m}$ in diameter. The accuracy with which it can measure $R_{\mathrm{g}, 0}$ is otherwise comparable to that of the conventional pyranometer method.

A solar spectrophotometer can be used to estimate $R_{\mathrm{g}, 0}$ to within 0.006 by averaging nearnormal beam hemispherical solar spectral reflectance $r_{\mathrm{b}, \mathrm{nn}}(\lambda)$ weighted with AM1GH solar spectral irradiance $i_{\mathrm{g}, 0}(\lambda)$. We recommend the incorporation of technique "E903G" for calculation of this value $R_{\mathrm{g}, 0}^{*}$ in the next revision of the ASTM E903 standard.

While the AM1.5 solar reflectance measured with version 5 of the Solar Spectrum Reflectometer can overestimate $R_{\mathrm{g}, 0}^{*}$ of a "cool colored" spectrally selective surface by as much as 0.08 , the SSRv6_AM1GH solar reflectance measured by version 6 of this instrument matches $R_{\mathrm{g}, 0}^{*}$ to within about 0.01 . We recommend upgrading existing units to version 6 .

\section{Acknowledgements}

This work was supported by the California Energy Commission (CEC) through its Public Interest Energy Research Program (PIER) and by the Assistant Secretary for Renewable Energy under Contract No. DE-AC02-05CH11231. The authors wish to thank PIER manager Chris Scruton and former CEC Commissioner Arthur Rosenfeld for their support and advice. We thank Charlie Moore of Devices \& Services (Dallas, TX) for characterization of the Solar Spectrum Reflectometer, and Kevin Stone for assistance in the development of method E1918A. We also thank James Dunn of the Ferro Corporation and Michelle Vondran of BASF Industrial Coatings for supplying test materials.

\section{References}

[1] Ronnen Levinson, Hashem Akbari, and Paul Berdahl. Measuring solar reflectance-Part I: defining a metric that accurately predicts solar heat gain. Submitted to Solar Energy-please 
update this reference for us, 2010.

[2] ASTM. ASTM E1918-06: Standard test method for measuring solar reflectance of horizontal and low-sloped surfaces in the field. Technical report, American Society for Testing and Materials, West Conshohocken, PA, 2006.

[3] Robert Siegel and John Howell. Thermal Radiation Heat Transfer, chapter 2. Taylor \& Francis, 4th edition, 2002.

[4] A. Lester and D.R. Myers. A method for improving global pyranometer measurements by modeling responsivity functions. Solar Energy, 80(3):322 - 331, 2006.

[5] Hashem Akbari, Ronnen Levinson, and Stephanie Stern. Procedure for measuring the solar reflectance of flat or curved roofing assemblies. Solar Energy Materials 8 Solar Cells, 82:648$655,2008$.

[6] ASTM. ASTM E903-96: Standard test method for solar absorptance, reflectance, and transmittance of materials using integrating spheres. Technical report, American Society for Testing and Materials, West Conshohocken, PA, 1996.

[7] ASTM. ASTM E891-87 (1992): Tables for terrestrial direct normal solar spectral irradiance for air mass 1.5. Technical report, American Society for Testing and Materials, West Conshohocken, PA, 1987.

[8] K. Emery, D. Myers, and S. Kurtz. What is the appropriate reference spectrum for characterizing concentrator cells? In Conference Record of the Twenty-Ninth IEEE Photovoltaic Specialists Conference, pages 841-843, New Orleans, Louisiana, May 20 - 24 2002. Institute of Electrical and Electronics Engineers, Inc. (IEEE). Online at http://www.nrel.gov/docs/ fy02osti/32284.pdf.

[9] ASTM. ASTM G173-03: Standard tables for reference solar spectral irradiances: direct normal and hemispherical on $37^{\circ}$ tilted surface. Technical report, American Society for Testing and Materials, West Conshohocken, PA, 2003. Spectra online at http://rredc.nrel.gov/solar/ spectra/am1.5. 
[10] C. A. Gueymard, D. Myers, and K. Emery. Proposed reference irradiance spectra for solar energy systems testing. Solar Energy, 73(6):443 - 467, 2002.

[11] Chris Gueymard. Recent developments in spectral solar radiation standards and modelling. Solar Spectrum (newsletter of the Resource Assessment Division of the American Solar Energy Society), 16(1):4 - 8, May 2003. Online at http://solardat.uoregon.edu/download/ Newslet/SolarSpectrum2003No1.pdf.

[12] ASHRAE. ANSI/ASHRAE 74-1988: Method of measuring solar-optical properties of materials. Technical report, American Society of Heating, Refrigerating, and Air-Conditioning Engineers, Atlanta, GA, 1988.

[13] ASTM. ASTM E891-82: Tables for terrestrial direct normal solar spectral irradiance for air mass 1.5. Technical report, American Society for Testing and Materials, West Conshohocken, PA, 1982.

[14] ASTM. ASTM E424-71 (2007): Standard test methods for solar energy transmittance and reflectance (terrestrial) of sheet materials. Technical report, American Society for Testing and Materials, West Conshohocken, PA, 2007.

[15] Parry Moon. Proposed standard solar-radiation curves for engineering use. Journal of the Franklin Institute, 230(5):583 - 617, 1940.

[16] Ronnen Levinson. AM1GH solar spectral irradiance. Online at http://coolcolors.lbl. gov/irradiance, 2010.

[17] Marilyne Andersen, Eleanor Stokes, Nicholas Gayeski, and Courtney Browne. Using digital imaging to assess spectral solar-optical properties of complex fenestration materials: A new approach in video-goniophotometry. Solar Energy, 84(4):549 - 562, 2010. International Conference CISBAT 2007.

[18] Devices \& Services. Devices \& Services Solar Spectrum Reflectometer. Online at http: //devicesandservices.com, 2010.

[19] M.P. Thekaekara. Data on incident solar energy. In M.P. Thekaekara, editor, The Energy Crisis and Energy from the Sun; Supplement to the Proceedings of the 20th Annual Meeting 
of the Institute of Environmental Sciences, pages 21-49, Washington, D.C., April 301974. Institute of Environmental Sciences.

[20] Charlie Moore. Personal communication, June 2002.

[21] Charlie Moore. The solar spectrum reflectometer (SSR). Technical Note 79-16, Devices \& Services, Dallas, TX, 1979. Online at http://devicesandservices.com/TechNotes/TN79-16. pdf.

[22] Charlie Moore. Personal communication, October 2008. 


\section{Nomenclature}

\section{English symbols}

$a_{j} \quad$ detector $j$ response multiplier

$c_{j} \quad$ detector $j$ reflectance weight

$D_{j} \quad$ detector $j$ reflectance

$F \quad$ view factor

$F_{j} \quad$ detector $j$ integrated response

$f_{j} \quad$ detector $j$ spectral response

$g \quad$ solar spectral response

$I_{\mathrm{i}} \quad$ incident solar irradiance

$I_{\mathrm{r}} \quad$ reflected solar irradiance

$i_{\mathrm{g}} \quad$ global spectral irradiance

$i_{\mathrm{g}, 0} \quad$ AM1GH spectral irradiance

$N \quad$ near-infrared reflectance

$R \quad$ solar reflectance

$R_{\mathrm{g}} \quad$ global solar reflectance

$R_{\mathrm{g}, 0} \quad$ AM1GH solar reflectance

$R_{\mathrm{g}, 0}^{*} \quad$ E903_AM1GH solar reflectance

$R_{\mathrm{E} 1918}$ solar reflectance measured with conventional pyranometer method ASTM E1918

$R_{\mathrm{E} 1918 \mathrm{~A}} \quad$ solar reflectance measured with alternative pyranometer method E1918A

$r_{\mathrm{b}, \mathrm{n}} \quad$ normal-incidence beam-hemispherical spectral reflectance

$r_{\mathrm{b}, \mathrm{nn}} \quad$ near-normal beam-hemispherical spectral reflectance

$S \quad$ solar reflectance

$V \quad$ visible reflectance

$Y_{j} \quad$ detector $j$ signal

$z \quad$ solar zenith angle

\section{Greek symbols}

$\gamma \quad$ surface solar azimuth angle

$\Delta S$ difference in solar reflectance

$\Delta I_{\mathrm{r}} \quad$ white-black difference in reflected solar irradiance

$\theta$ incidence angle

$\lambda \quad$ wavelength

$\Sigma \quad$ surface tilt angle

$\phi \quad$ target diameter 


\section{Initialisms}

AM air mass

AM0BN air mass 0 beam normal

AM1BN (clear-sky) air mass 1 beam normal

AM1DH (clear-sky) air mass 1 diffuse horizontal

AM1GH (clear-sky) air mass 1 global horizontal

AM1.5GT- (clear-sky) air mass 1.5 global tilt opposing sun

$\mathrm{B} n \quad$ (spectral) band $n$

E1918 (ASTM Standard) E1918

E1918A (alternative pyranometer method) E1918A

E891BN (ASTM Standard) E891 beam normal

E903 (ASTM Standard) E903

E903_n average of solar spectral reflectance weighted with solar spectral irradiance $n$

G173BN (ASTM Standard) G173 beam normal

G173GT (ASTM Standard) G173 global tilt

LST local standard time

NIR near infrared

SSR Solar Spectrum Reflectometer

SSRv5 Solar Spectrum Reflectometer version 5

SSRv5_n SSRv5 solar reflectance output corresponding to solar spectral irradiance $n$

SSRv6 Solar Spectrum Reflectometer version 6

SSRv6_n SSRv6 solar reflectance output corresponding to solar spectral irradiance $n$

SSRv6_V5n SSRv6 solar reflectance output emulating SSRv5 solar reflectance output $n$ 


\begin{tabular}{|c|c|c|c|c|}
\hline & L1v5 & L2v5 & L3v5 & L4v5 \\
\hline AM0 & 0.341 & 0.328 & 0.236 & 0.095 \\
AM1 & 0.313 & 0.407 & 0.239 & 0.041 \\
AM1.5 & 0.343 & 0.416 & 0.210 & 0.031 \\
AM2 & 0.313 & 0.449 & 0.211 & 0.027 \\
AM1GH_aux & 0.251 & 0.395 & 0.239 & 0.115 \\
AM1BN_aux & 0.275 & 0.420 & 0.217 & 0.087 \\
AM1DH_aux & 0.044 & 0.185 & 0.422 & 0.348 \\
AM0BN_aux & 0.308 & 0.353 & 0.197 & 0.141 \\
G173GT_aux & 0.267 & 0.427 & 0.220 & 0.086 \\
G173BN_aux & 0.287 & 0.440 & 0.206 & 0.067 \\
E891BN_aux & 0.318 & 0.452 & 0.168 & 0.062 \\
\hline
\end{tabular}

Table 1: Detector weights $c_{j}$ used to calculate SSRv5 native solar reflectances AM0, AM1, AM1.5 and AM2 and SSRv5 auxiliary solar reflectances AM1GH_aux, AM1BN_aux, AM1DH_aux, AM0BN_aux, G173BT_aux and G173BN_aux from SSRv5 detector reflectances L1v5...L4v5 (a.k.a. $\left.D_{1} \ldots D_{4}\right)$. 


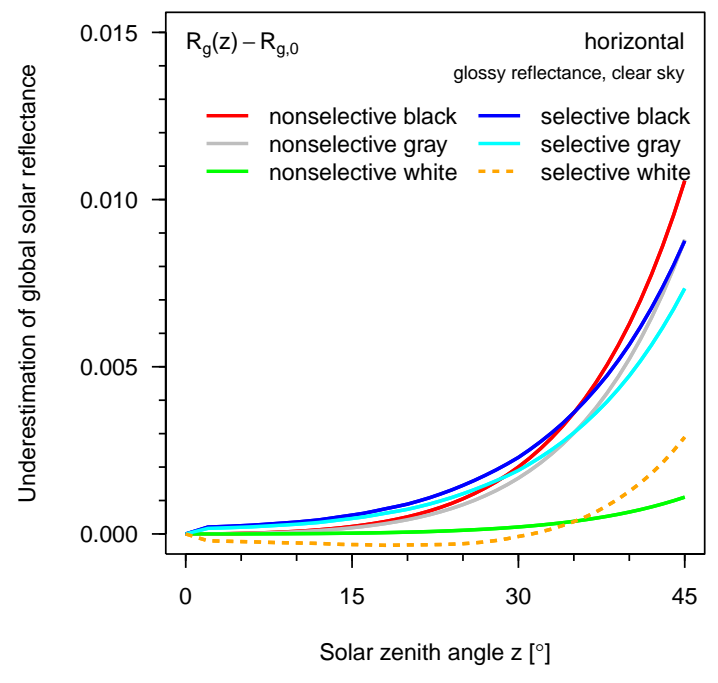

Figure 1: Variation with solar zenith angle angle $z\left(0-45^{\circ}\right)$ of the amount by which AM1GH solar reflectance $R_{\mathrm{g}, 0}$ underestimates the global solar reflectance $R_{\mathrm{g}}(z)$ of a glossy horizontal surface.

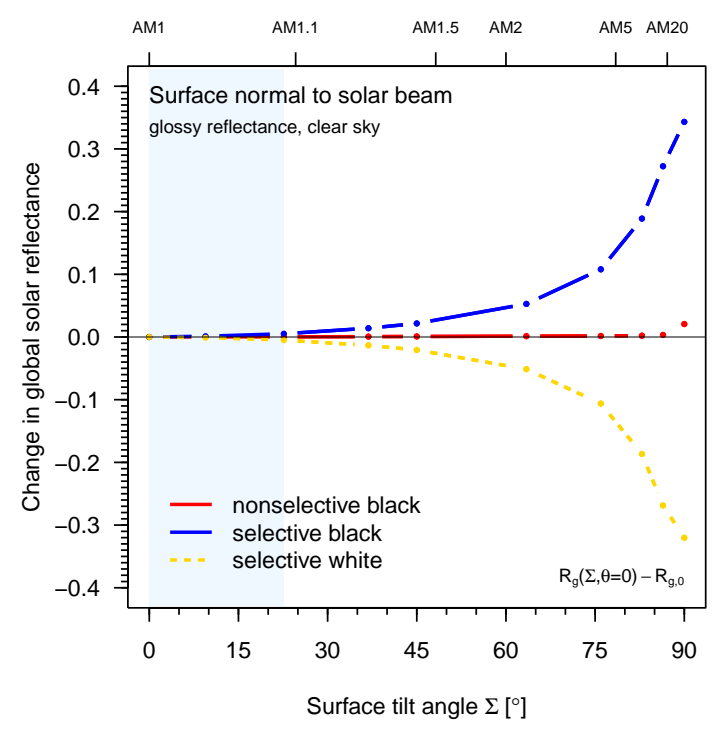

Figure 2: Variation with tilt angle $\left(0-90^{\circ}\right)$ of normal-incidence global solar reflectance of a glossy surface. Tilts up to 5:12 [23] are shaded. Results for a matte surface are very similar.
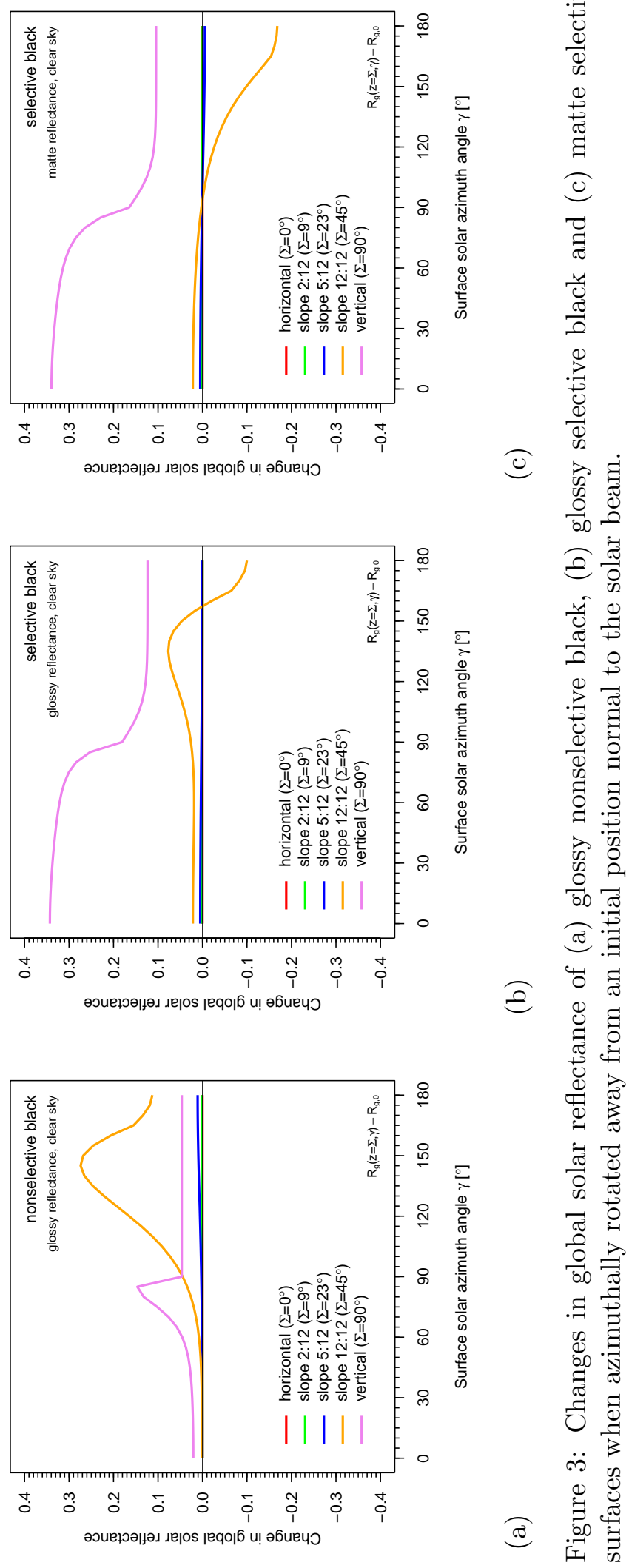

April 28, 2010

.




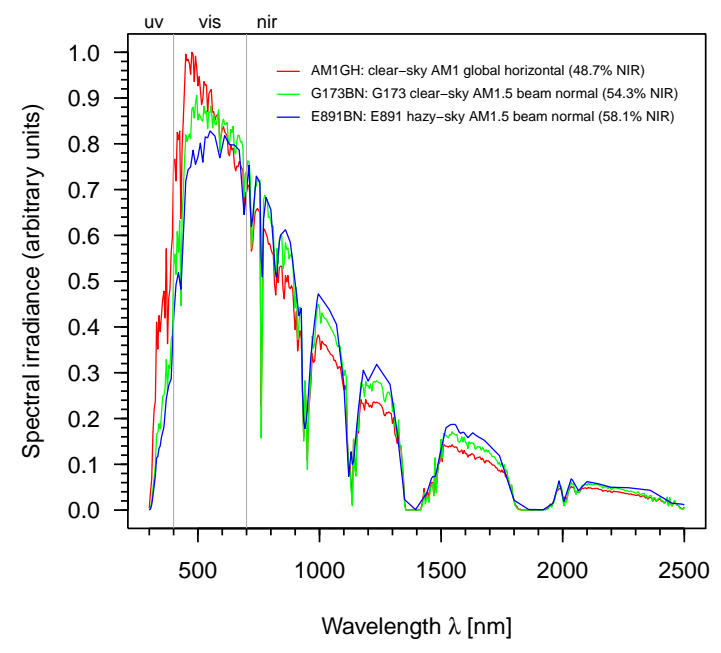

Figure 4: Comparison of AM1GH, G173BN and E891BN solar spectral irradiances.

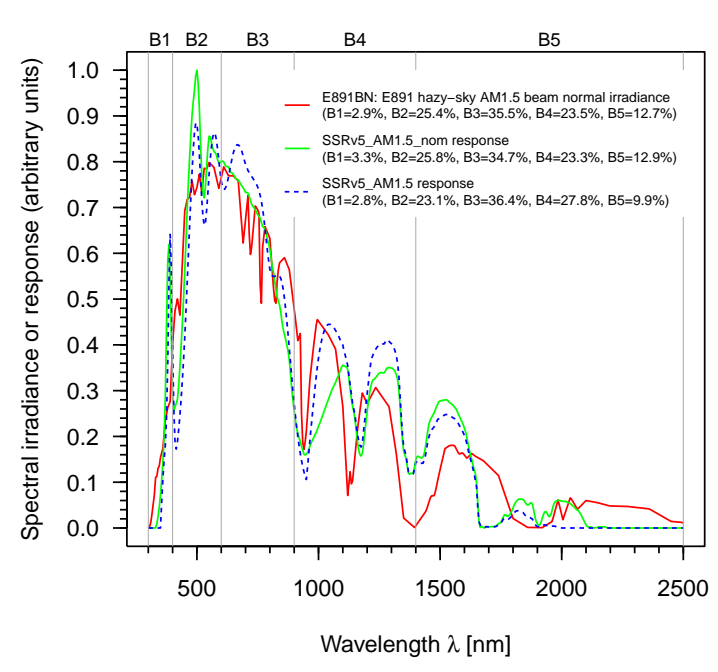

Figure 5: Approximation of the E891BN solar spectral irradiance by the nominal and actual AM1.5 solar spectral responses of version 5 of Solar Spectrum Reflectometer. B1...B5 are the spectral bands in which the power fractions of the nominal response are matched to those of the irradiance.

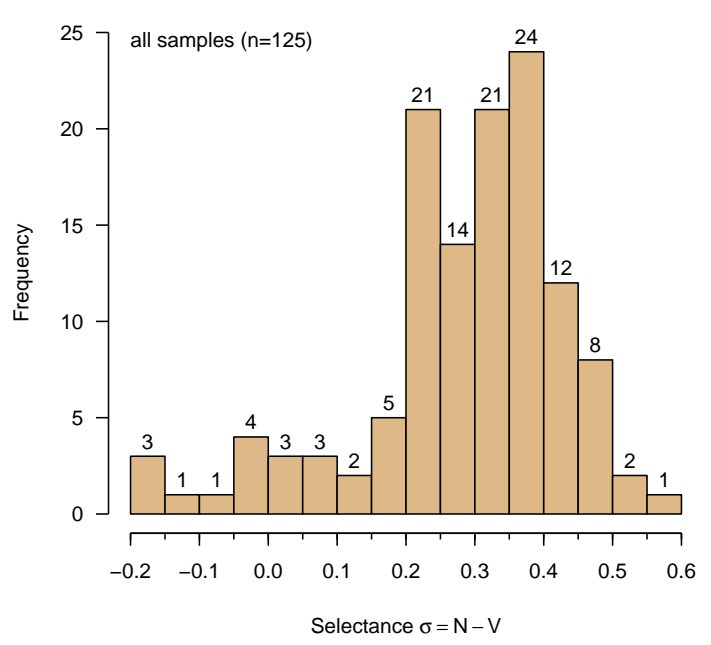

Figure 6: Selectances of all 125 samples.

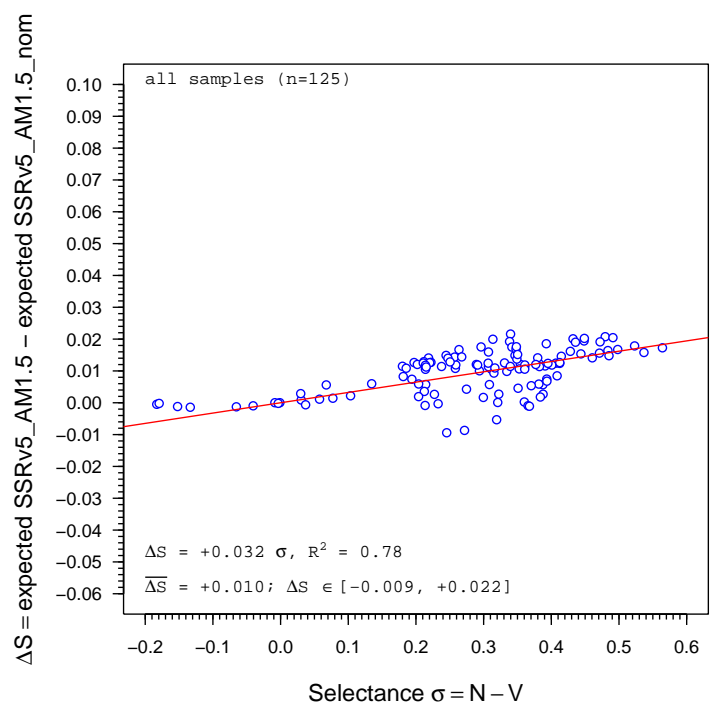

Figure 7: Variation with selectance $\sigma$ of the amount $\Delta S$ by which the solar reflectance expected from the actual SSRv5_AM1.5 spectral response exceeds the solar reflectance expected from the nominal SSRv5_AM1.5 spectral response. 


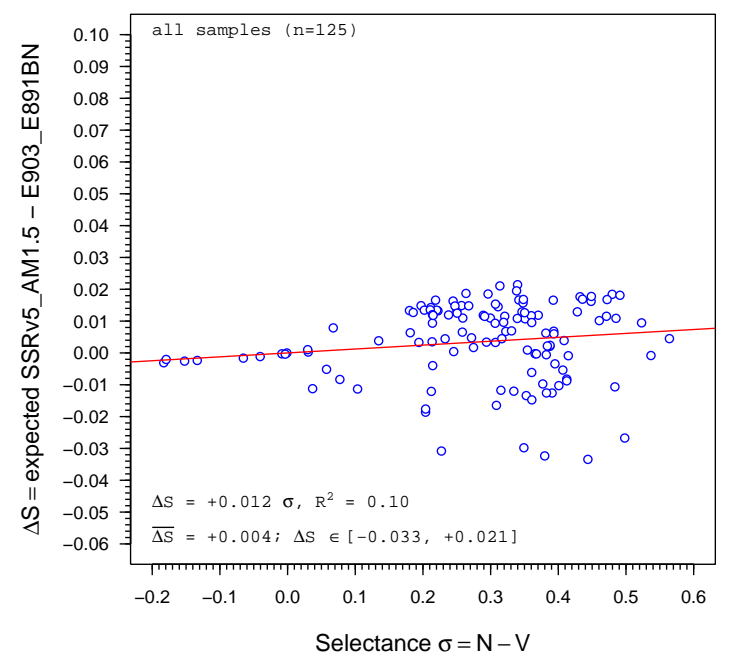

Figure 8: Variation with selectance $\sigma$ of the amount $\Delta S$ by which the solar reflectance expected from the actual SSRv5_AM1.5 spectral response exceeds E903_E891BN solar reflectance.

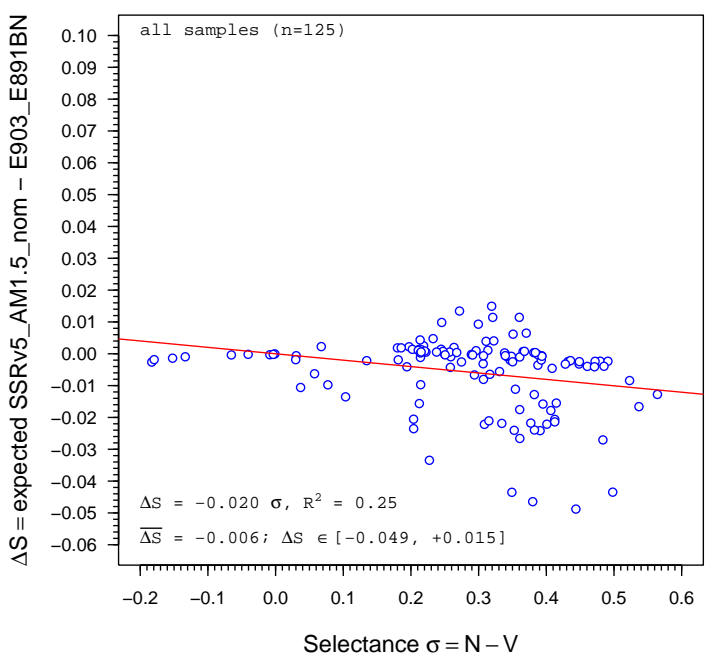

Figure 9: Variation with selectance $\sigma$ of the amount $\Delta S$ by which the solar reflectance expected from the nominal SSRv5_AM1.5 spectral response exceeds E903_E891BN solar reflectance.

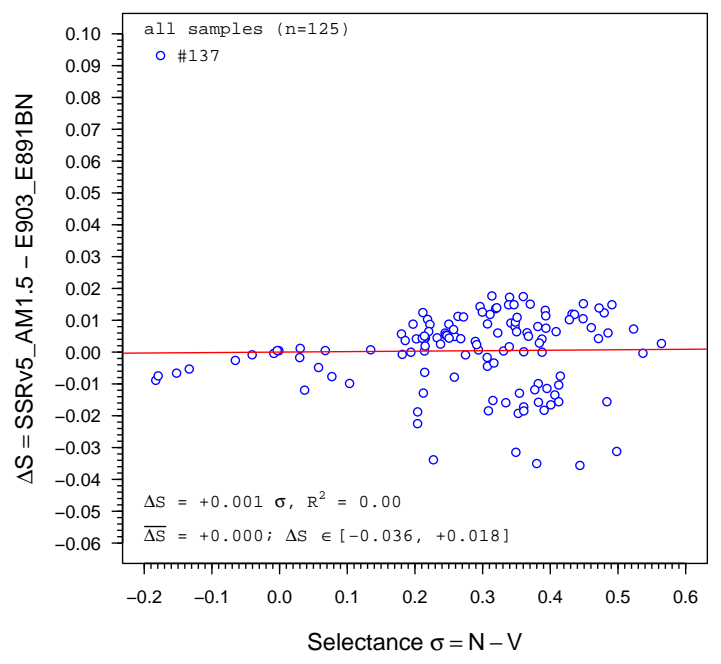

Figure 10: Variation with selectance $\sigma$ of the amount $\Delta S$ by which SSRv5_AM1.5 solar reflectance measured with instrument \#137 exceeds E903_E891BN solar reflectance.

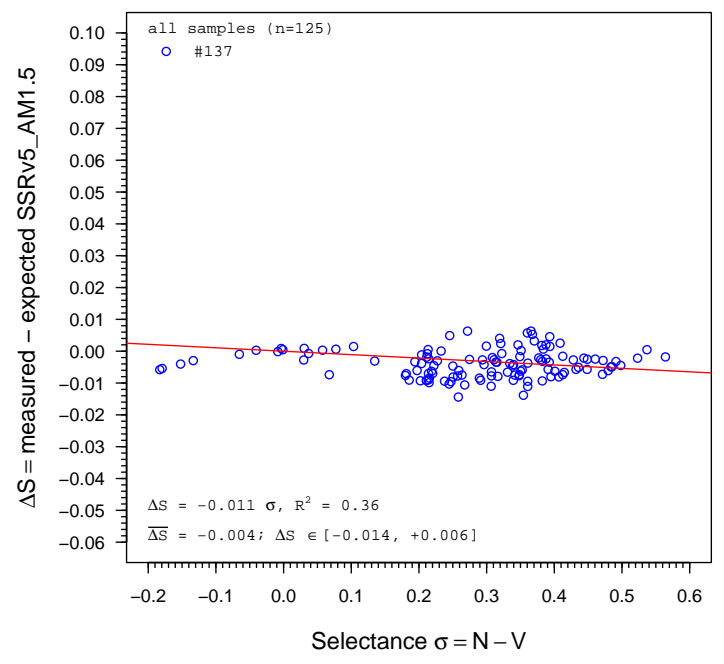

Figure 11: Variation with selectance $\sigma$ of the amount $\Delta S$ by which SSRv5_AM1.5 solar reflectance measured with instrument \#137 exceeds the SSRv5_AM1.5 solar reflectance expected from the actual SSRv5_AM1.5 solar spectral response. 


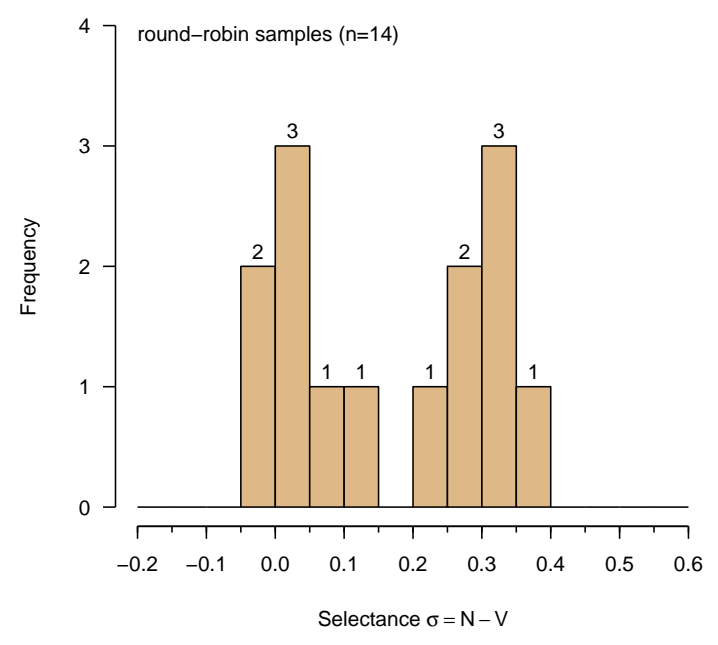

Figure 12: Spectral selectances of 14 samples circulated in round robin.

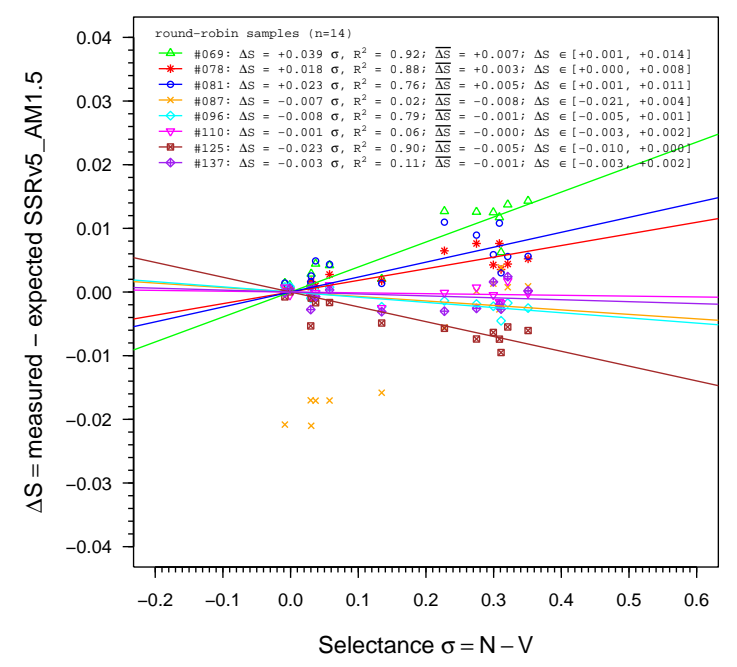

Figure 13: Variation with selectance $\sigma$ of the amount $\Delta S$ by which the SSRv5_AM1.5 solar reflectances measured with eight different instruments exceed the SSRv5_AM1.5 solar reflectance expected from the actual SSRv5_AM1.5 solar spectral response.

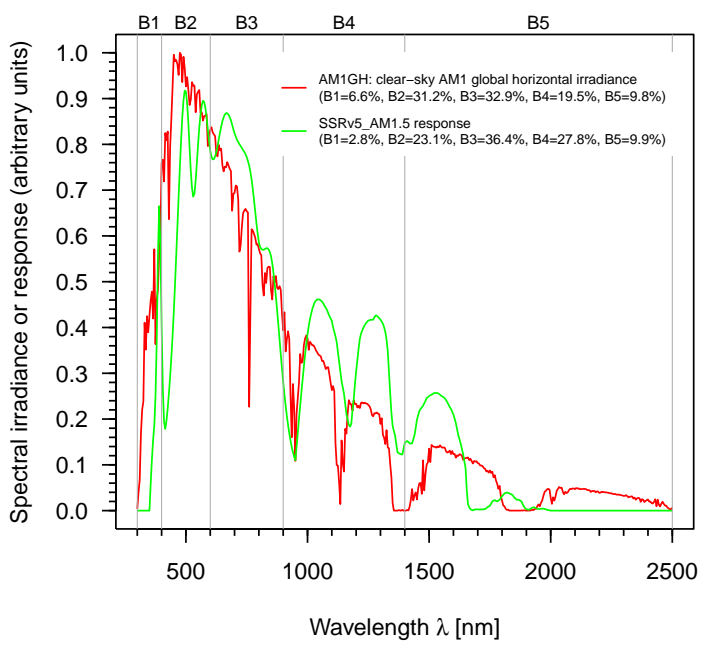

Figure 14: Approximation of the AM1GH solar spectral irradiance by the actual SSRv5_AM1.5 solar spectral response.

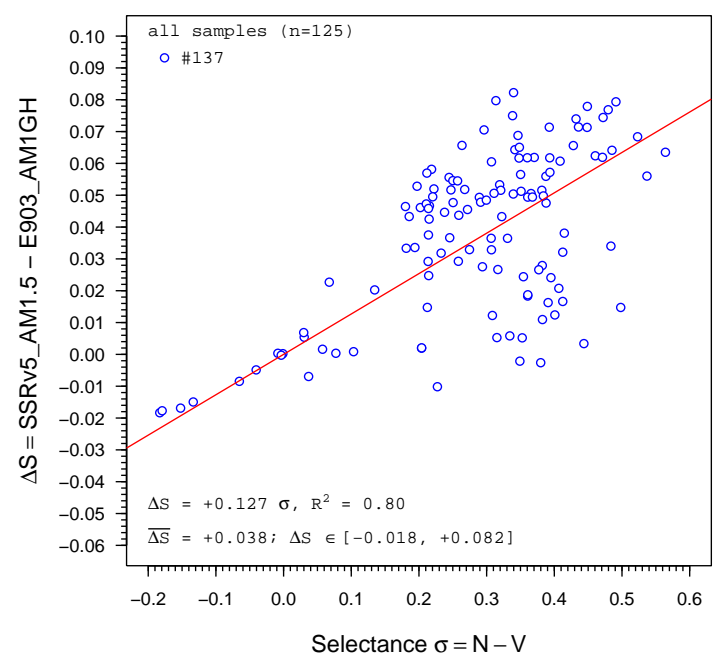

Figure 15: Variation with selectance $\sigma$ of the amount $\Delta S$ by which SSRv5_AM1.5 solar reflectance measured with instrument \#137 exceeds E903_AM1GH solar reflectance. 

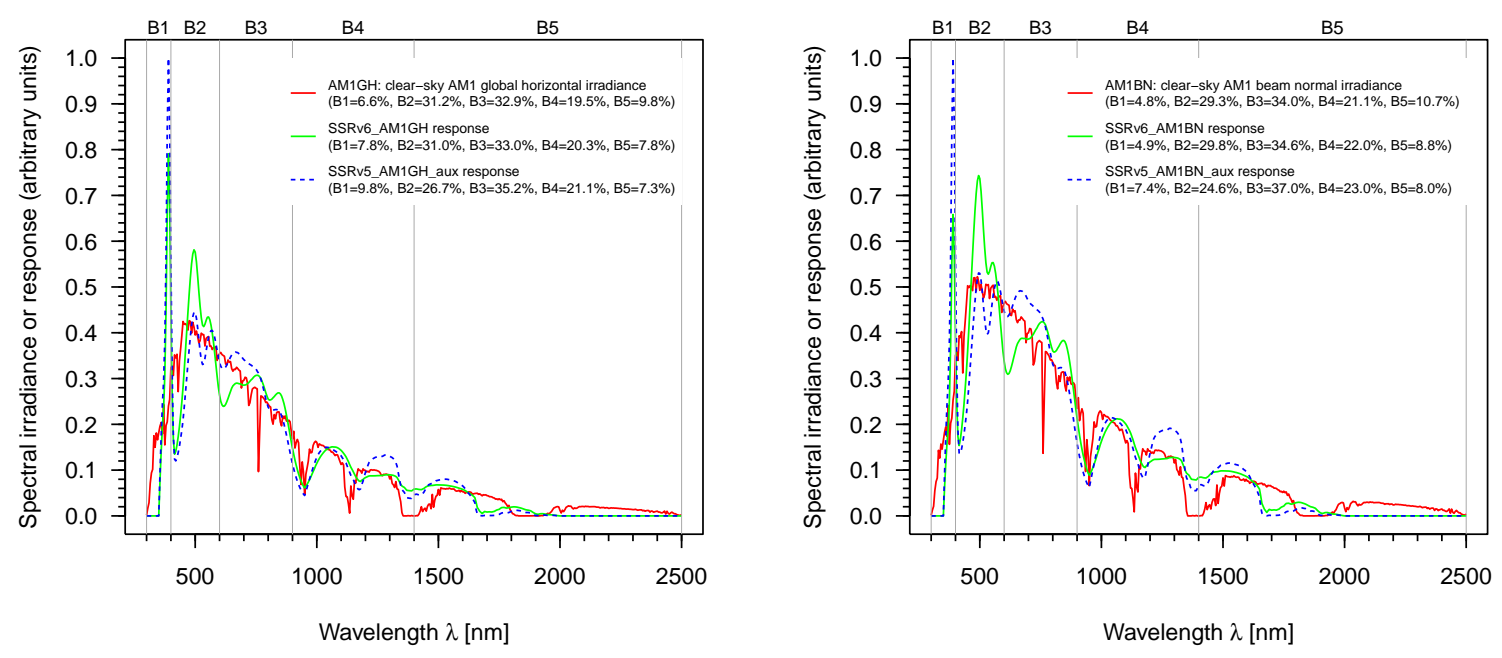

(a)
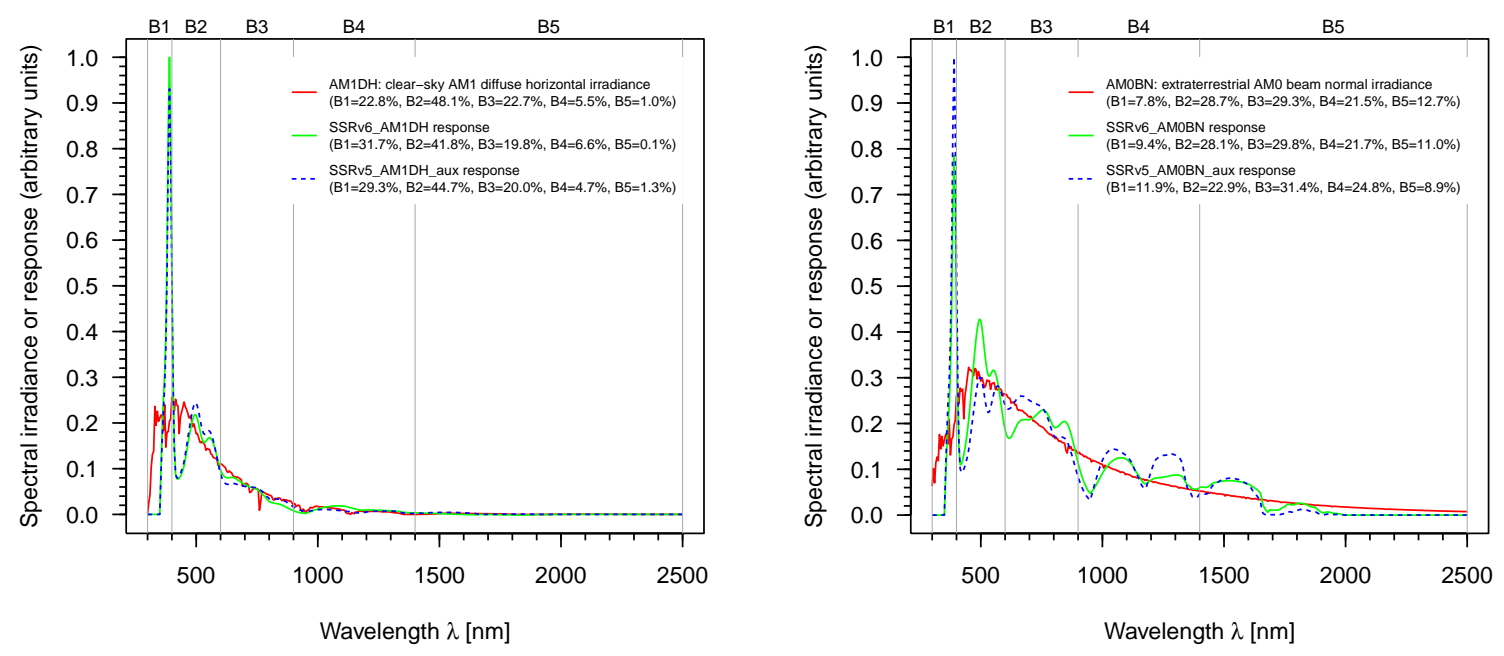

(c)

(d)

Figure 16: Approximation of solar spectral irradiance $n$ by the SSRv6_n and SSRv5_n_aux solar spectral responses, shown for (a) AM1GH, (b) AM1BN, (c) AM1DH, (d) AM0BN, (e) G173GT, (f) G173BN and (g) E891BN. 

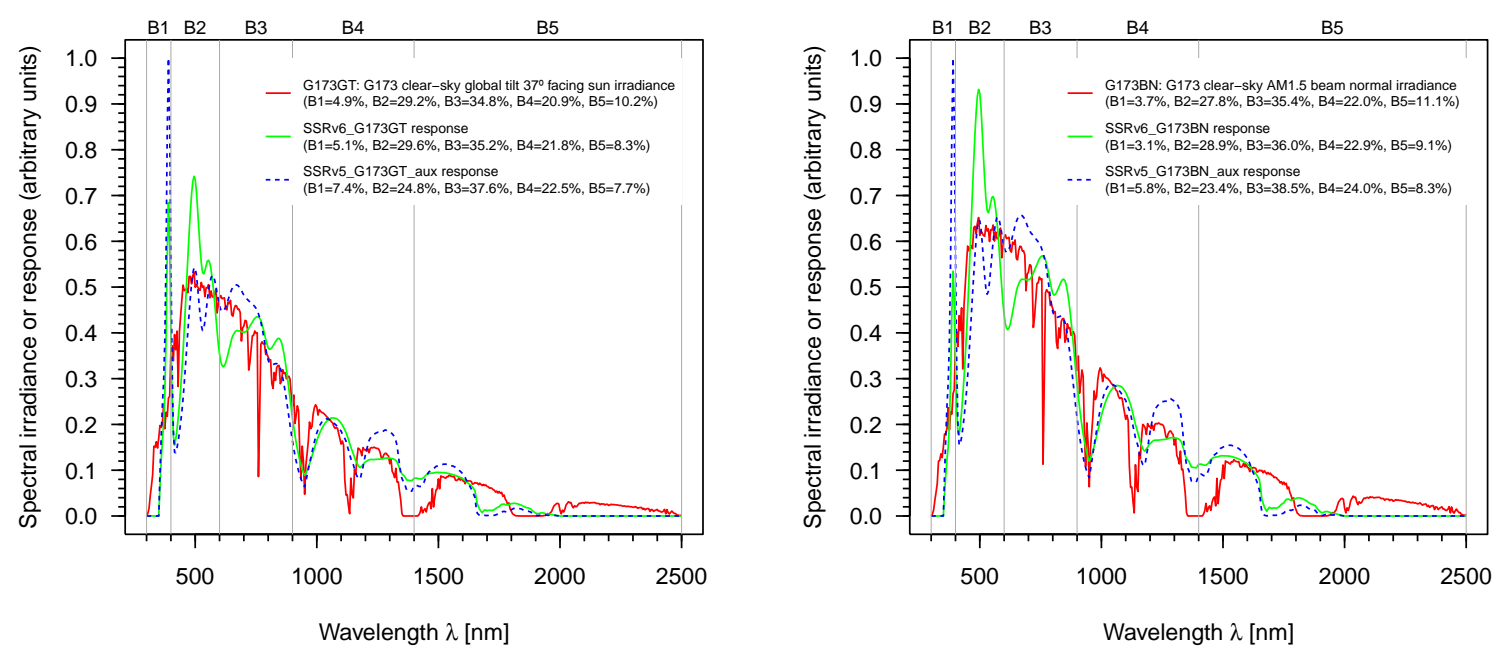

(e)

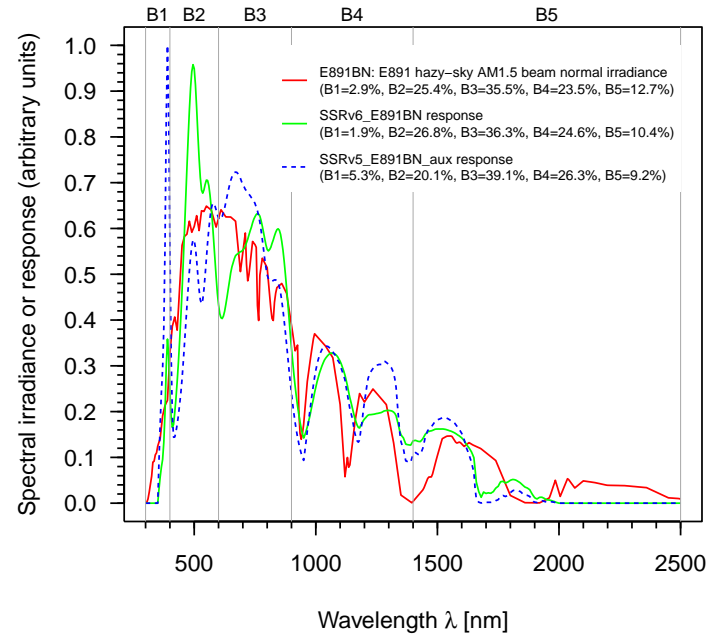

(g)

Figure 16: continued. 


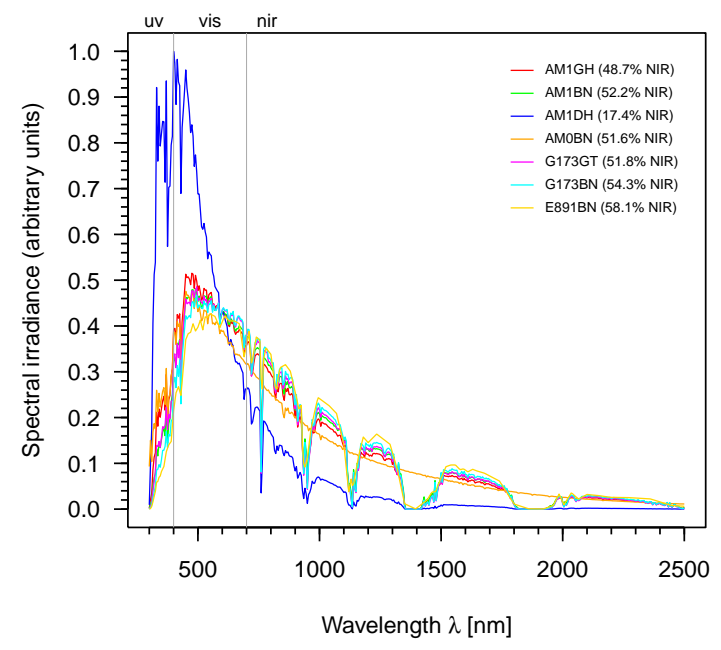

Figure 17: Contrast between the shape of the AM1DH solar spectral irradiance and those of AM1GH, AM1BN, AM0BN, G173GT, G173BN and E891BN. 


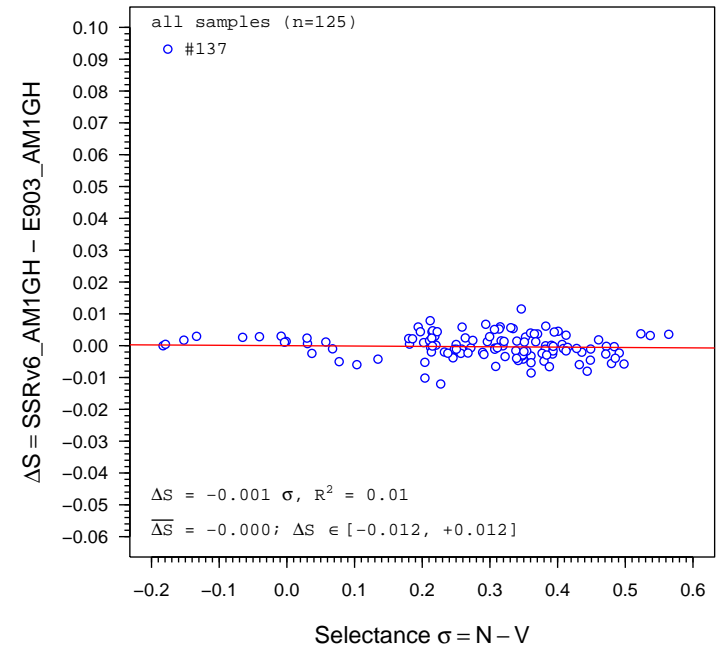

(a)

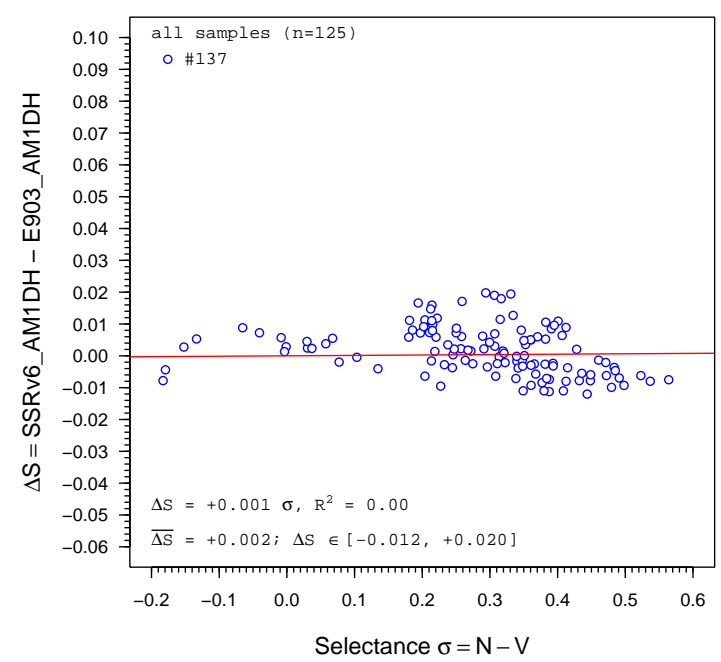

(c)

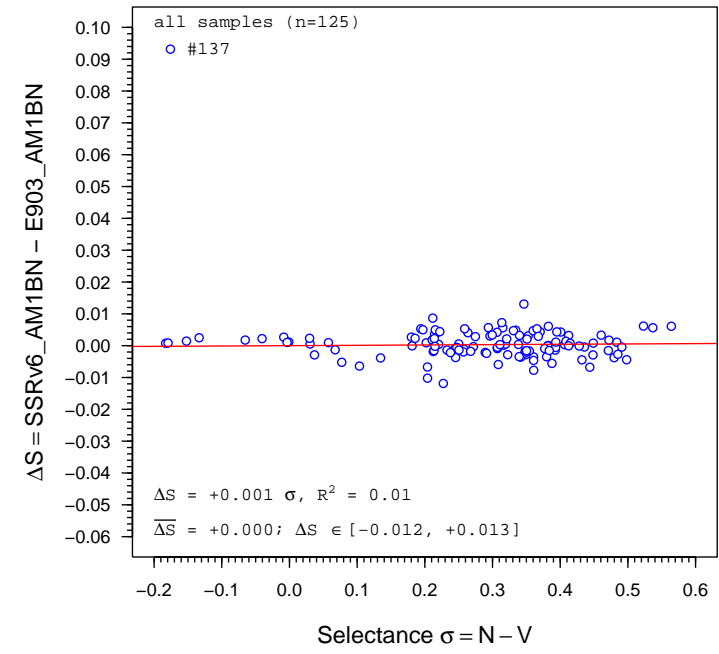

(b)

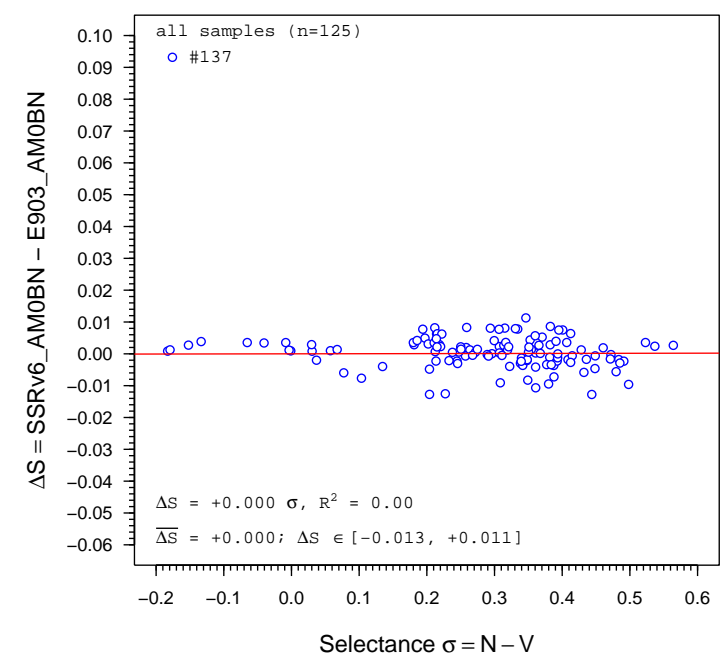

(d)

Figure 18: Variation with selectance $\sigma$ of the amount $\Delta S$ by which SSRv6_n solar reflectance measured with instrument \#137 exceeds E903_n solar reflectance, shown for (a) AM1GH, (b) AM1BN, (c) AM1DH, (d) AM0BN, (e) G173GT, (f) G173BN and (g) E891BN. 

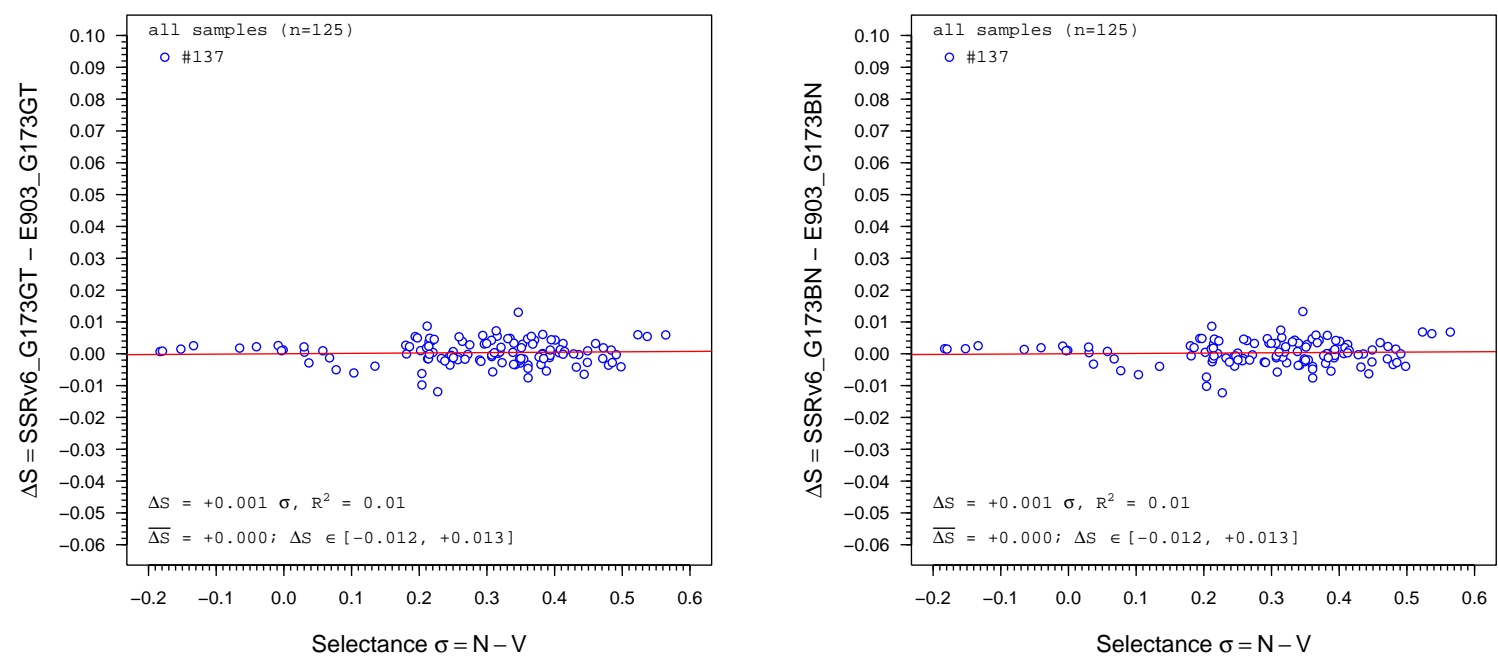

(e)

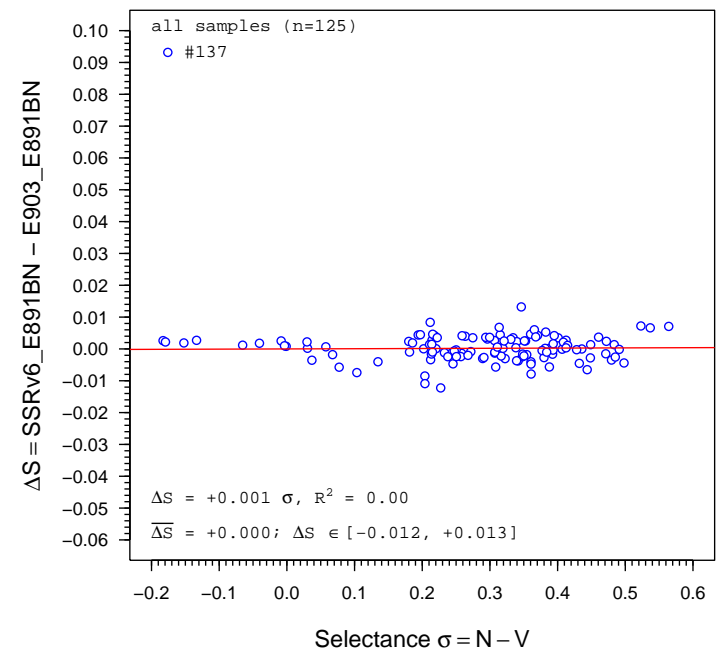

(g)

Figure 18: continued. 


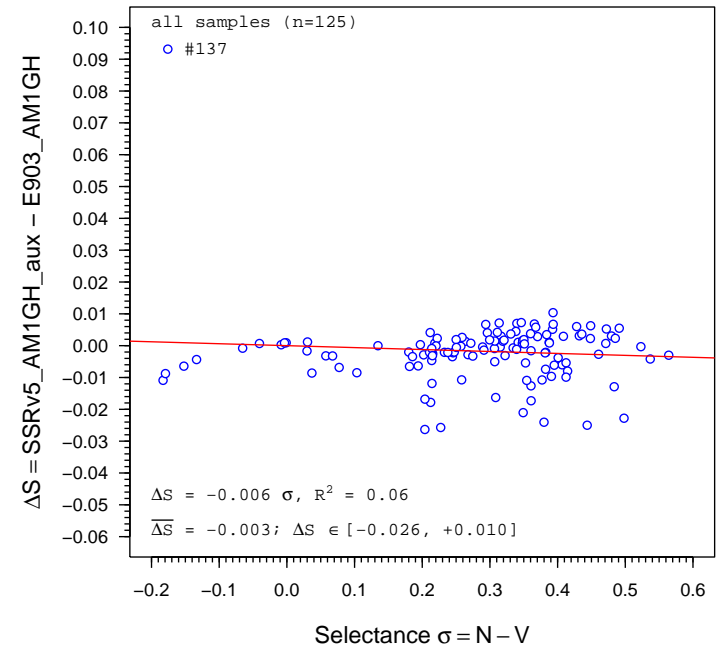

(a)

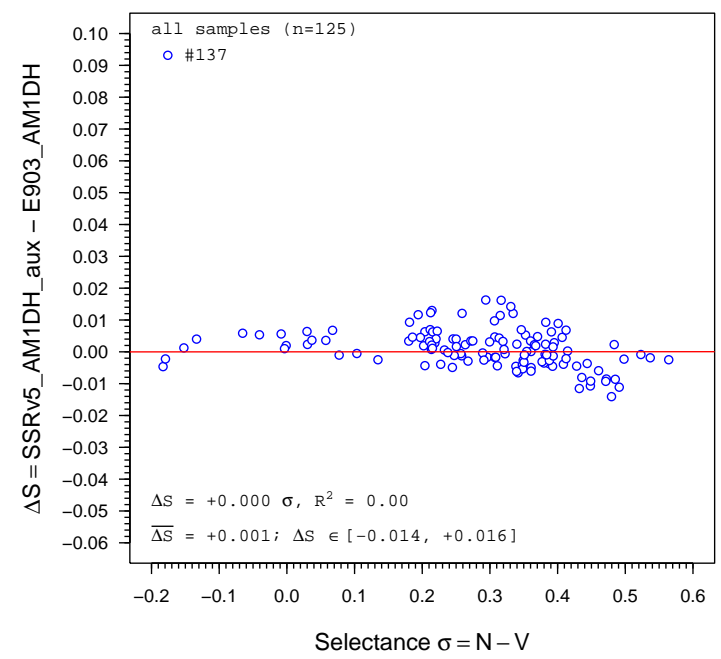

(c)

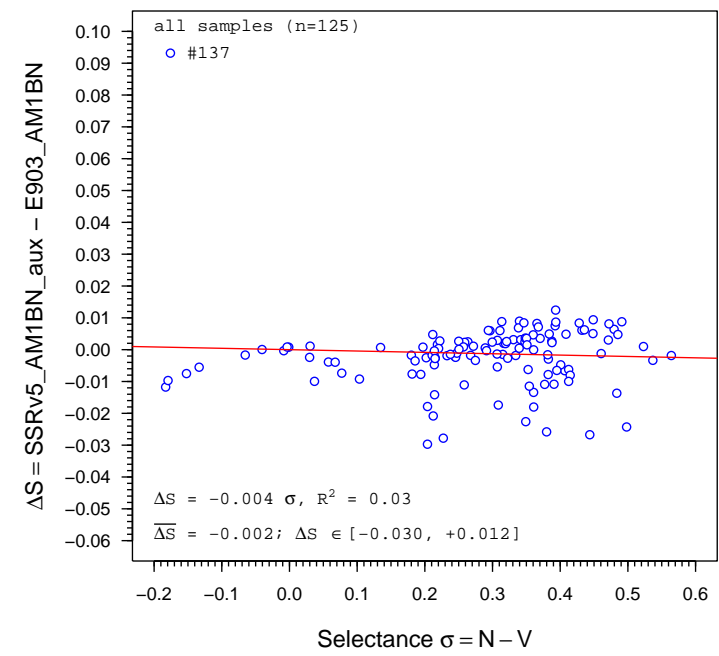

(b)

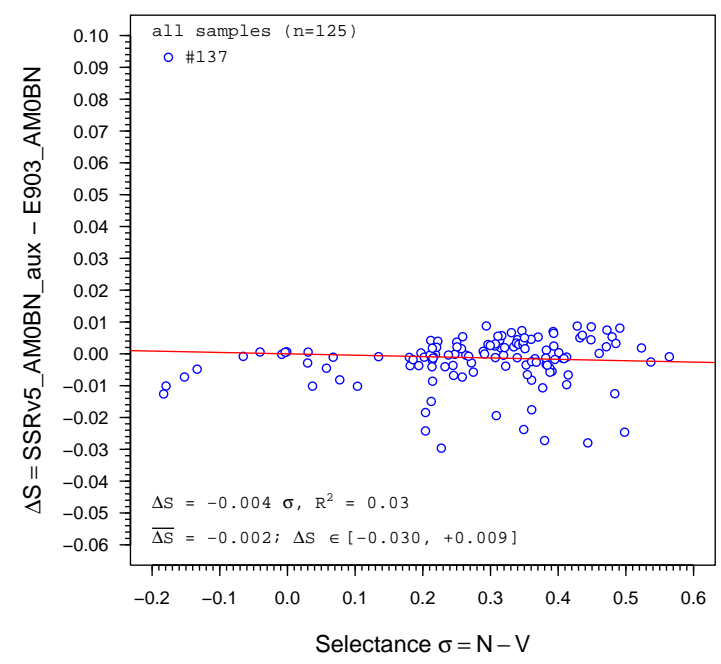

(d)

Figure 19: Variation with selectance $\sigma$ of the amount $\Delta S$ by which SSRv5_n_aux solar reflectance measured with instrument \#137 exceeds E903_n solar reflectance, shown for (a) AM1GH, (b) AM1BN, (c) AM1DH, (d) AM0BN, (e) G173GT, (f) G173BN and (g) E891BN. 

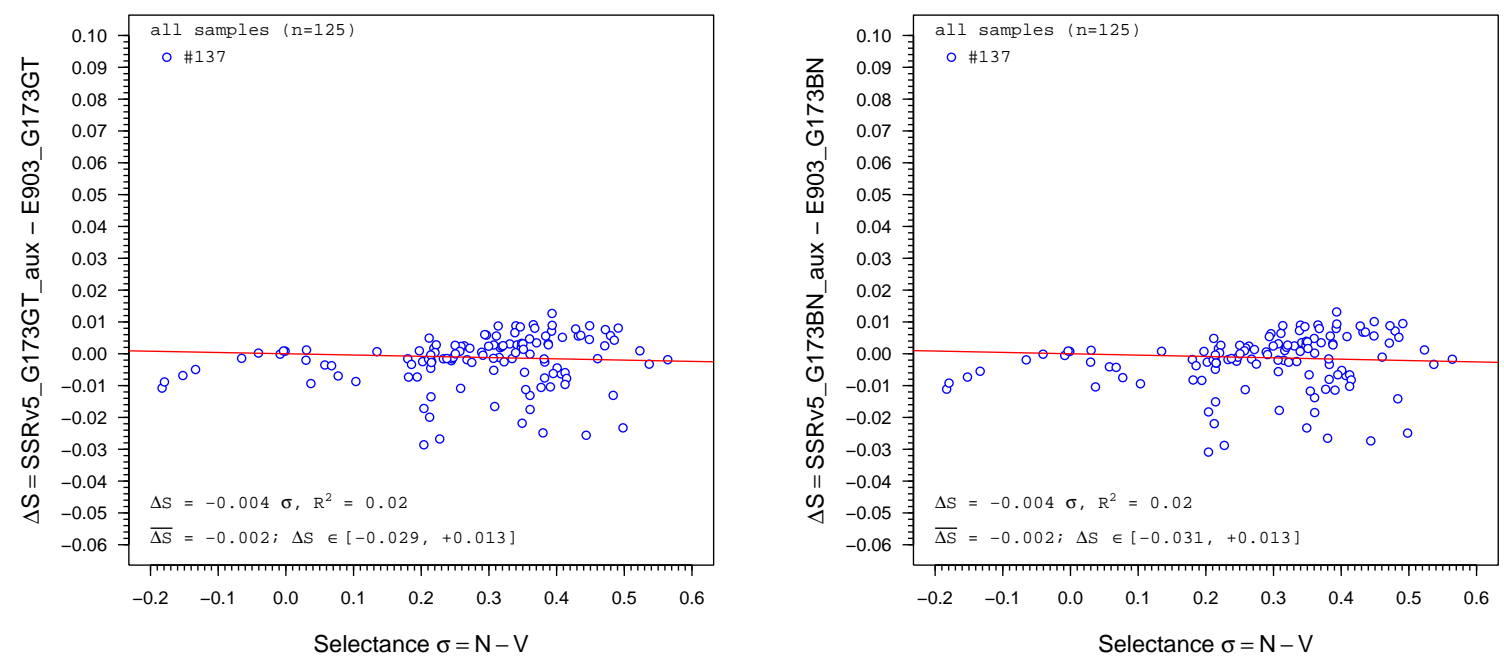

(e)

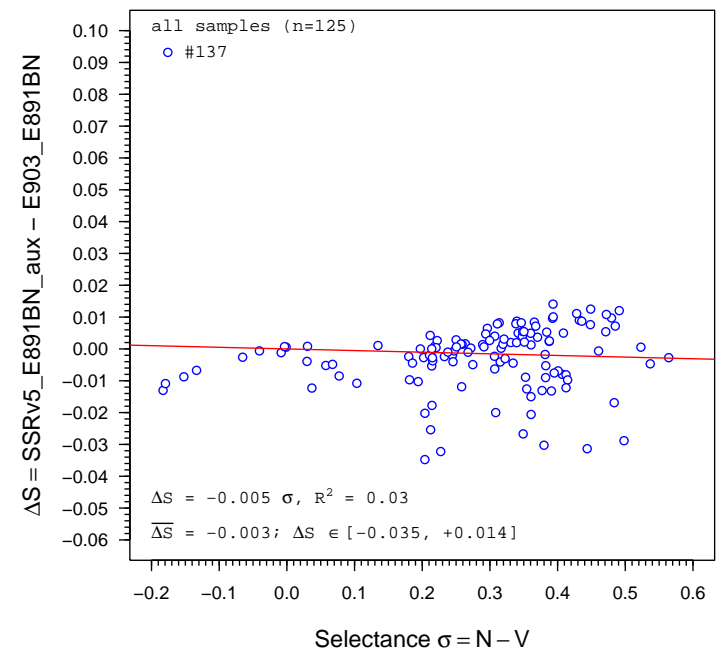

(g)

Figure 19: continued. 


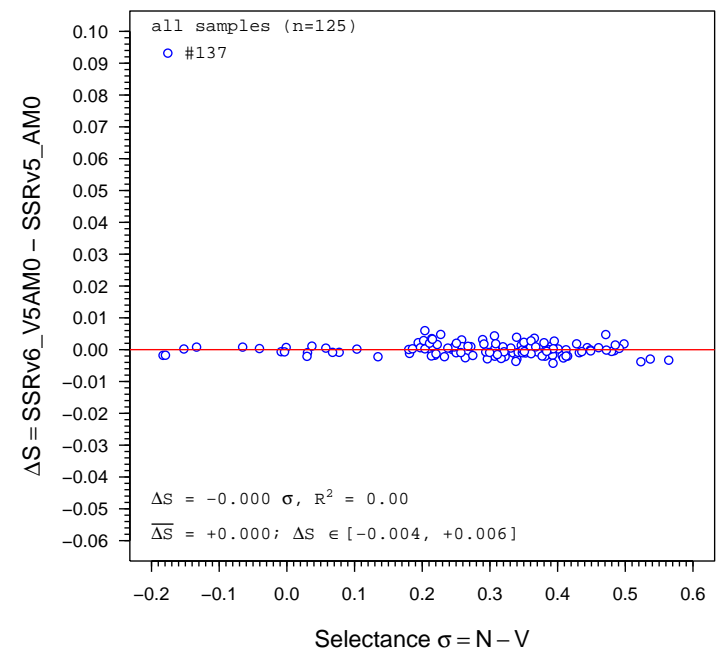

(a)

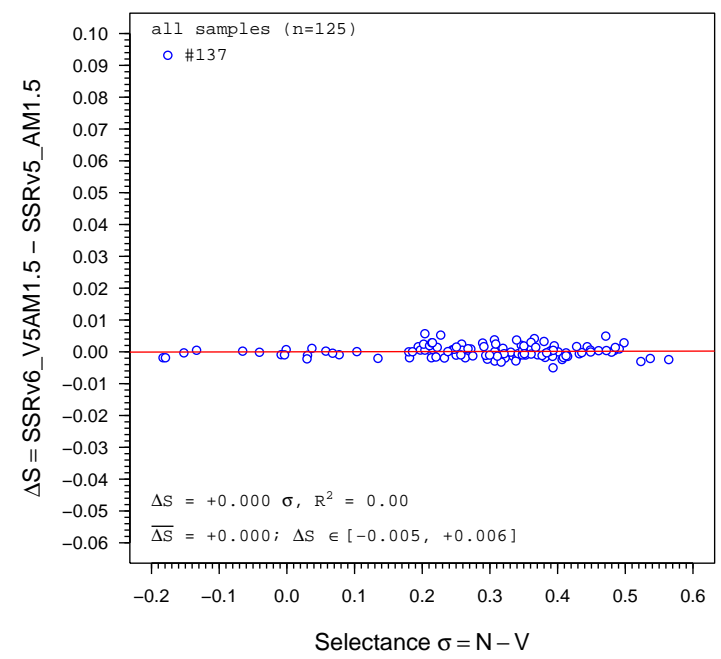

(c)

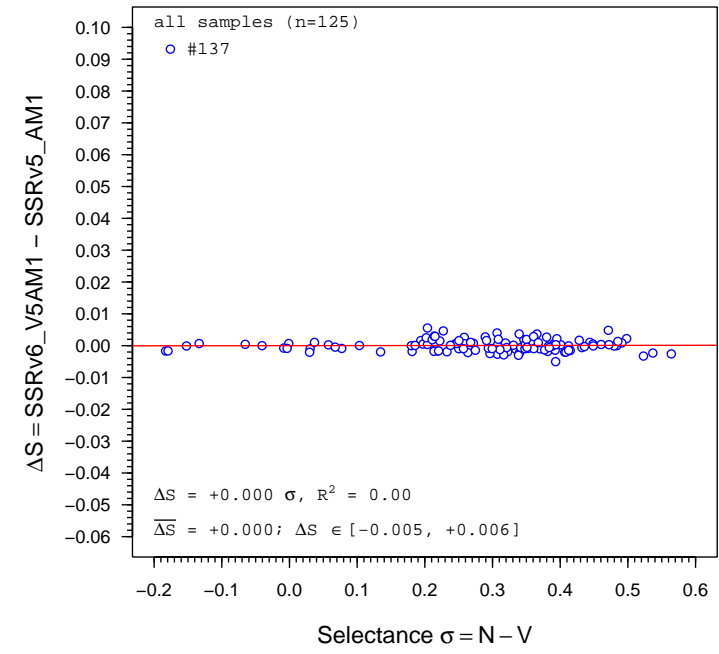

(b)

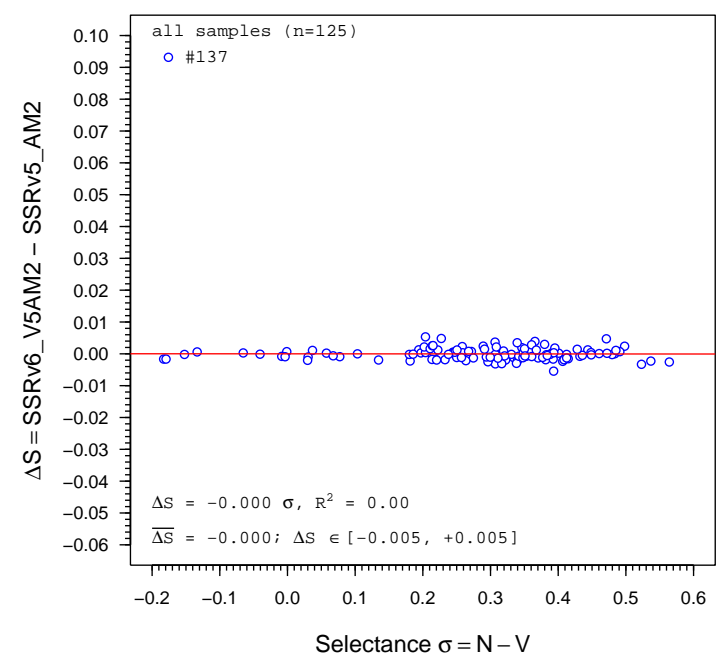

(d)

Figure 20: Variation with selectance $\sigma$ of the amount $\Delta S$ by which SSRv6_V5n solar reflectance measured with instrument \#137 exceeds SSRv5_n solar reflectance, shown for (a) AM0, (b) AM1, (c) AM1.5 and (d) AM2. 